\title{
Current approaches to the management of new-onset ulcerative colitis
}

This article was published in the following Dove Press journal:

Clinical and Experimental Gastroenterology

9 May 2014

Number of times this article has been viewed

\section{Renée Marchioni Beery' Sunanda Kane ${ }^{2}$ \\ 'Division of Gastroenterology and Hepatology, University of Connecticut Health Center, Farmington, CT, USA; ${ }^{2}$ Division of Gastroenterology and Hepatology, Mayo Clinic, Rochester, MN, USA}

\begin{abstract}
Ulcerative colitis (UC) is an idiopathic, inflammatory gastrointestinal disease of the colon. As a chronic condition, UC follows a relapsing and remitting course with medical maintenance during periods of quiescent disease and appropriate escalation of therapy during times of flare. Initial treatment strategies must not only take into account current clinical presentation (with specific regard for extent and severity of disease activity) but must also take into consideration treatment options for the long-term. The following review offers an approach to new-onset UC with a focus on early treatment strategies. An introduction to the disease entity is provided along with an approach to initial diagnosis. Stratification of patients based on clinical parameters, disease extent, and severity of illness is paramount to determining course of therapy. Frequent assessments are required to determine clinical response, and treatment intensification may be warranted if expected improvement goals are not appropriately reached. Mild-to-moderate UC can be managed with aminosalicylates, mesalamine, and topical corticosteroids with oral corticosteroids reserved for unresponsive cases. Moderate-to-severe UC generally requires oral or intravenous corticosteroids in the short-term with consideration of long-term management options such as biologic agents (as initial therapy or in transition from steroids) or thiopurines (as bridging therapy). Patients with severe or fulminant UC who are recalcitrant to medical therapy or who develop disease complications (such as toxic megacolon) should be considered for colectomy. Early surgical referral in severe or refractory UC is crucial, and colectomy may be a life-saving procedure. The authors provide a comprehensive evidence-based approach to current treatment options for new-onset UC with discussion of long-term therapeutic efficacy and safety, patient-centered perspectives including quality of life and medication compliance, and future directions in related inflammatory bowel disease care.
\end{abstract}

Keywords: ulcerative colitis, inflammatory bowel disease, new-onset treatment, acute management

\section{Introduction}

Ulcerative colitis (UC) is a chronic, idiopathic, inflammatory disease limited to the colon. Inflammation involves the rectum in a majority (95\%) of patients and extends proximally in a continuous and circumferential fashion. ${ }^{1}$ Disease may involve the entire colorectum (termed pancolitis) or only parts of it as manifested in cases of proctitis (limited to the rectum) or left-sided colitis (involving the sigmoid colon with or without descending colon). A few patients may develop limited terminal ileal involvement (backwash ileitis) that can be difficult to differentiate from Crohn's ileocolitis.

UC presents clinically as abdominal pain and bloody diarrhea commonly associated with symptoms of rectal urgency and tenesmus. Extraintestinal manifestations are common. The diagnosis is suspected based on clinical history after exclusion of
Correspondence: Renée Marchioni Beery Division of Gastroenterology and Hepatology, 263 Farmington Avenue, Farmington, CT 06030-1845, USA

Tel +l 8606793158

Fax+l 8606793159

Email marchioni@uchc.edu 
infectious and noninfectious etiologies ${ }^{2}$ and is supported by both characteristic endoscopic findings and histologic confirmation. ${ }^{1}$ Endoscopic features include continuous mucosal ulceration from the rectum with erythema, friability, and loss of typical vascular pattern. Histologic features include crypt architectural distortion, cryptitis, and crypt abscesses. Mucosal inflammation is generally superficial, although patients with severe UC may develop transmural inflammation and deep colonic ulcerations that increase the risk of toxic megacolon. Severe symptoms are less commonly seen with left-sided colitis and proctitis.

As a chronic disease, UC is characterized by periods of relapse and remission with flares of disease activity occurring spontaneously or provoked by certain aggravating factors such as intercurrent illness, antibiotic use, or nonadherence with medical therapy. Achieving control over active disease and maintaining patients in steroid-free remission are the optimal goals of therapy. This review will provide an introduction to $\mathrm{UC}$ with focus on treatment strategies for the management of new-onset cases.

\section{Epidemiology}

Inflammatory bowel disease (IBD), encompassing both UC and Crohn's disease (CD), has been increasing in incidence and prevalence in various regions of the globe with the highest rates in North America and Europe. ${ }^{3}$ A recent study using a large, regionally diverse population of commercially insured individuals in the US revealed a prevalence of 263 (95\% confidence interval [CI] 260-266) per 100,000 for adult UC as of 2009. It is estimated that approximately 593,000 Americans have UC and that there has been a slight increase in the prevalence of IBD over time. ${ }^{4}$ Regional variation in the US has been reported for adult UC, with a lower prevalence in the South and West as compared with the Northeast and Midwest. ${ }^{4}$ In two large prospective cohorts of US women, the incidence of IBD was significantly lower among females living in southern versus northern latitudes, and residence beyond age 30 years was more strongly associated with risk. $^{5}$

The onset of IBD has a bimodal distribution with a higher peak in the younger population aged 15-30 years; $50 \%$ of patients afflicted by IBD are diagnosed before age 35 years. ${ }^{6,7}$ Most studies reveal a male:female ratio of about 1:1 across all ages. ${ }^{8}$ Standardized disease-related mortality in UC is similar to that of the general population. Additionally, the cause of death in UC is overall comparable to that of the general population and is related to $\mathrm{UC}$ or its associated illnesses in approximately $17 \%$ of cases. ${ }^{9}$
The standardized incidence ratio of colorectal cancer in IBD patients is 1.7 (95\% CI 1.2-2.2) and increases to 6.4 (95\% CI 2.4-17.5) in patients with extensive colitis. ${ }^{10}$ Despite recent therapeutic advances, morbidity remains high and can significantly impact a patient's quality of life (QOL).

\section{Economic burden}

The US economic burden of UC is increasing and has been estimated at approximately US\$8.1-14.9 billion annually with total direct costs of about $\$ 3.4-8.6$ billion. ${ }^{11,12}$ US health care-associated treatment costs for UC totaled approximately $\$ 2.1$ billion in $2008 .{ }^{12}$ The estimated yearly per-patient direct medical cost of UC ranges from \$6,217-11,477 with hospitalizations accounting for roughly $41 \%-55 \%$; indirect costs comprise about one-third of the total US cost. ${ }^{11}$ From 1998-2004, the overall hospitalization rate for UC was 10.8 per 100,000 with approximately 214,498 inpatient admissions for UC and an average hospital length of stay of 6.8 days. ${ }^{13}$ Inpatient charges related to UC rose over time to an estimated annual total of $\$ 945$ million nationally. ${ }^{13}$ A significant increase in the number and incidence of US hospitalizations has been reported in the pediatric IBD population with 22,328 children carrying a hospital discharge diagnosis of $\mathrm{UC}$ and 7,127 children hospitalized for UC (incidence 15.2-26.0 cases per 100,000; $P<0.001$ ) from 2000-2009; this has also been linked to a significant increase in IBD-related complications (such as need for surgery, concurrent infection, and obstruction) and comorbid disease burden (at least three comorbidities). ${ }^{14}$ Direct costs, hospitalizations, and surgeries appear to increase with severity of illness. ${ }^{11}$

\section{Pathogenesis}

The exact pathogenesis of UC is unknown; the underlying mechanisms appear complex and multifaceted. Mucosal inflammation is thought to result from an aberrant immune response to intestinal microbiota arising in genetically predisposed individuals with additional contributions of lifestyle and environmental factors among others. ${ }^{15}$ Multiple genes ${ }^{16}$ and cellular pathways have been implicated as well. ${ }^{17}$

\section{Initial approach in consideration of new-onset UC}

Diagnosis and management of new-onset UC, particularly in the setting of severe cases, requires a comprehensive and multidisciplinary approach. The primary focus is to stratify disease activity in order to determine course of medical therapy and 
possible role for surgery. Assessment of disease extent should be pursued as a prognostic indication; pancolonic involvement is associated with higher rates of failed medical therapy, disease-related complications, and colectomy. ${ }^{18,19}$

\section{Disease classification and assessment of activity}

UC cases are organized into mild, moderate, and severe disease categories largely based on clinical symptoms and objective data (Table 1). ${ }^{20}$ Such classification helps guide medical management and treatment options that can then be tailored based on patient response and progression. While there is no single standardized disease index for $\mathrm{UC}$, the Truelove and Witts Index is often used in defining and quantifying cases of severe $\mathrm{UC} .^{20}$

The Mayo Clinic Index (or Disease Activity Index) is a scoring system for assessing disease activity in UC. This scoring can be used for initial evaluation and for monitoring of response to therapy. The Mayo Clinic Index score ranges from zero to twelve, taking into account stool frequency, rectal bleeding, endoscopic findings, and physician's global assessment. ${ }^{21,22}$ Scores from two to five indicate mild disease, while scores from six to twelve indicate moderate-to-severe disease. The endpoint definition of remission has been described as a Mayo Clinic Index score of two or less with no individual subscore greater than one. Each individual patient serves as a control in establishing the degree of abnormality in stool frequency. The daily bleeding score is based on the most severe bleeding episodes of the day. The physician's global assessment includes the patient's daily recall of abdominal discomfort and general sense of wellbeing in addition to other observations such as physical assessment and performance status. ${ }^{22}$

\section{History and physical examination}

Patients should be queried regarding current symptoms such as abdominal pain and distension, bowel habits (frequency, nocturnal movements, blood in stool), urgency or tenesmus, presence of extraintestinal manifestations, nausea, vomiting, fever, and weight loss. Potential inciting factors include cessation of cigarette smoking or use of concurrent medications such as nonsteroidal anti-inflammatories or oral contraceptives. ${ }^{1}$ Recent hospitalization/stress, use of antibiotics or narcotics, travel, and dietary history should be considered and can be elicited during clinical history.

Close physical examination with attention to vital signs, mentation, and clinical status is paramount to rule out sepsis or hypovolemia. Abdominal inspection (particularly for distension or hernias) should precede auscultation for bowel sounds and palpation for abdominal tenderness, organomegaly, rebound, or guarding. A rectal examination with investigation of the perianal region, stool, and rectal vault should always be performed. Examination of the skin, eyes, and joints can determine the presence of extraintestinal manifestations.

\section{Differential considerations}

Exclusion of alternate etiologies and concomitant infection are crucial steps in initial evaluation. ${ }^{2}$ Differential diagnoses that can mimic UC include diverticulitis, appendicitis, bowel obstruction, infectious or medication-induced colitis, ischemic colitis, or vasculitis. Stool microbiologic testing

Table I Categorization of clinical parameters and disease severity in ulcerative colitis

\begin{tabular}{|c|c|c|c|c|}
\hline \multirow[t]{2}{*}{ Clinical parameters } & \multicolumn{4}{|c|}{ Disease severity } \\
\hline & Mild & Moderate & Severe & Fulminant \\
\hline \multicolumn{5}{|l|}{ Stool variables } \\
\hline Number per day & $<4$ & $\geq 4$ & $>6$ & $>10$ \\
\hline Stool appearance & 土blood & 土blood & +blood & Continuous rectal bleeding \\
\hline \multicolumn{5}{|l|}{ Clinical features } \\
\hline Temperature $\left({ }^{\circ} \mathrm{C}\right)$ & Normal & Normal or $>37.5$ & $>37.5$ & $>37.5$ \\
\hline Heart rate & Normal & Normal or tachycardic & Tachycardic & Tachycardic \\
\hline Clinical signs & $\begin{array}{l}\text { No signs of } \\
\text { toxicity }\end{array}$ & Minimal signs of toxicity & $\begin{array}{l}\text { Increasing signs of } \\
\text { toxicity; abdominal pain }\end{array}$ & $\begin{array}{l}\text { Toxic-appearing; abdominal } \\
\text { pain/distension }\end{array}$ \\
\hline \multicolumn{5}{|l|}{ Objective data } \\
\hline $\begin{array}{l}\text { Erythrocyte sedimentation } \\
\text { rate (mm/hour) }\end{array}$ & $\leq 30$ (normal) & Normal or elevated & $>30$ (elevated) & $>30$ (elevated) \\
\hline Hemoglobin & Normal & Normal or low & Anemia ( $<75 \%$ of normal value) & Anemia requiring transfusion \\
\hline Radiographic imaging & & & $\begin{array}{l}\text { Colonic air and bowel wall } \\
\text { edema with thumbprinting }\end{array}$ & Colonic dilation \\
\hline
\end{tabular}

Note: Data from Truelove and Witts. ${ }^{20}$ 
with bacterial culture and ova and parasite assay should be undertaken with inclusion of specific stool assays for Escherichia coli 0157:H7 and Clostridium difficile toxin. $C$. difficile colitis in IBD is rising in incidence and prevalence ${ }^{19,23}$ and carries an increased short-term and 5-year mortality in hospitalized UC patients; an increased rate of all-cause hospital readmissions has been observed, although colectomy rates appear to be unaffected. ${ }^{24}$ Viral and amebic infections should also be considered. Cytomegalovirus infection can appear very similarly to active UC (both clinically and endoscopically) and occurs in up to $34 \%$ of patients with acute severe colitis and $36 \%$ of patients in the steroid-refractory subgroup. ${ }^{25}$ A positive cytomegalovirus immunoglobulin $\mathrm{G}$ requires colonic biopsies with histologic examination (hematoxylin/eosin staining) searching for inclusion bodies as the most specific indicator for active viral replication. ${ }^{25}$ Concern should exist for opportunistic infections in immunocompromised hosts (human immunodeficiency virus, post-transplant, steroid-dependent or immunosuppressed patients); expansion of differentials should be undertaken in predisposed individuals (tuberculosis in endemic populations, recent travelers).

\section{Laboratory investigation}

Initial laboratory evaluation should include a complete blood count with differential, comprehensive metabolic profile including liver function panel, erythrocyte sedimentation rate, and C-reactive protein.

\section{Endoscopy}

Colonoscopy with biopsy is the first-line investigation in diagnosing UC and assessing disease extent and severity. Visual inspection can identify alternate or concomitant pathology including pseudomembranous colitis associated with $C$. difficile infection or ischemic colitis. Deep ulcerations could indicate the presence of cytomegalovirus colitis, $\mathrm{CD}$, or severe UC. The general safety of colonoscopy in patients with acute colitis has been documented ${ }^{26}$ without an increased major complication rate based on disease activity. ${ }^{27}$ However, a higher perforation risk during colonoscopy has been documented in hospitalized IBD patients versus controls without IBD ( $1 \%$ versus $0.6 \%$, respectively; $P<0.0001$ ) with no significant difference between UC and CD patients. ${ }^{28}$ Additionally, a higher perforation risk in IBD patients has been reported in association with increased endoscopic disease severity and steroid use. ${ }^{29}$ Thus, caution is advised in cases of severe colonic ulceration or distension to avoid precipitation of a perforation or toxic megacolon. The benefits of completing a full endoscopic examination to the terminal ileum must be weighed against the risks to the patient on an individual basis. Early flexible sigmoidoscopy with biopsy, perhaps coupled with abdominal imaging, may provide a safer alternative to full colonoscopy in selected cases. Endoscopy is generally contraindicated in the presence of toxic megacolon.

\section{Imaging}

Although imaging is not required for the diagnosis of UC, abdominal plain films should be considered in all patients presenting with colitis to exclude colonic dilation or free air. Abdominal radiography is usually normal in patients with mild-to-moderate disease but may identify proximal constipation, mucosal thickening ("thumbprinting") due to edema, or colonic dilation in severe cases. Computed tomography or magnetic resonance (MR) imaging of the abdomen/pelvis is indicated in patients demonstrating significant abdominal pain/distension, nausea, vomiting, fever, or leukocytosis.

In cases when conventional colonoscopy may not be completed or is not feasible due to the risk of complications such as perforation or toxic megacolon, a less invasive imaging modality may be considered. MR enterography is one such example and has advanced in recent years as a preferred imaging technique for providing a comprehensive view of intraluminal and extraluminal pathology without the use of ionizing radiation. In many North American sites, MR enterography has replaced gastrointestinal fluoroscopy and computed tomography enterography and has become a first-line imaging test for suspected or extant IBD. ${ }^{30}$ Similarly, MR colonography has shown high accuracy for assessment of disease activity and severity in UC using objective MR imaging parameters including colonic contrast uptake, mural edema, enlarged lymph nodes, and the presence of engorged perienteric vasculature (comb sign). ${ }^{31}$ As these MR findings correlate closely with endoscopy, this noninvasive study may be considered as an adjunct or alternative to colonoscopy in selected cases, particularly when colonoscopy is contraindicated or incomplete due to clinically severe illness.

\section{Hospitalization and initial inpatient management}

The risk of hospitalization in acute severe UC is $18 \%-25 \%$. $^{32}$ Although the incidence rate of hospitalizations has declined, ${ }^{8,34}$ the cumulative probability of first hospitalization 
for UC has increased, particularly with consecutive years of disease, and has recently been reported as $29.4 \%$ at 5 years (95\% CI $24.5 \%-34.1 \%), 38.7 \%$ at 10 years $(95 \%$ CI $33.1 \%-43.8 \%), 49.2 \%$ at 20 years $(95 \%$ CI $42.7 \%-55.2 \%)$, and $52.3 \%$ at 30 years (95\% CI $45.1 \%-59.7 \%) .{ }^{8}$ Early requirement both for hospitalization (hazard ratio 1.5; 95\% CI 1.02-2.4) and for corticosteroids (hazard ratio 1.8; 95\% CI 1.1-2.7) are independent predictors of hospitalization after illness of 90 days. ${ }^{8}$ Extensive colitis is predictive of future hospitalizations. ${ }^{8}$

Indications for hospitalization include severe disease, toxic megacolon, failure of outpatient medical therapy, profound extraintestinal manifestations, or complications related to IBD (such as thromboembolism) or its pharmacotherapy. Supportive inpatient management includes intravenous (IV) fluid administration, electrolyte and hemoglobin monitoring with repletion as needed, and IV corticosteroids if indicated. Anti-peristaltic and narcotic agents should be avoided, as they can potentially contribute to ileus, exacerbate colitis severity, and precipitate toxic megacolon. Patients should undergo early colonoscopy (typically within initial 48 hours) as the preferred endoscopic test to evaluate the full extent of disease and to obtain mucosal biopsies. As aforementioned, colonoscopy should be pursued with caution in cases of severe colonic ulceration or dilation to avoid precipitation of a perforation or toxic megacolon.

Consideration of medical options for acute severe UC should be undertaken, and early surgical consultation should be requested. Careful frequent examinations and assessments are required to determine the response to pharmacotherapy and to assess the need for additional rescue measures including colectomy. Approximately $40 \%-50 \%$ of patients hospitalized for UC will fail corticosteroid therapy ${ }^{32}$ so testing to facilitate forthcoming management should be pursued on admission. This includes testing for thiopurine methyltransferase enzyme activity for possible use of azathioprine (AZA) or 6-mercaptopurine; latent Mycobacterium tuberculosis (chest radiograph and purified protein derivative or QuantiFERON $^{\circledR}$-TB Gold [QIAGEN, Venlo, the Netherlands]) and hepatitis B (hepatitis B surface antigen, surface antibody, core antibody) for possible biologic agent; and serum cholesterol and magnesium levels for possible cyclosporine (CsA) or tacrolimus.

Careful monitoring for disease-related complications such as arterial or venous thromboembolism (VTE) is warranted. IBD is associated with a roughly 1.5- to 3.5fold increased risk of thromboembolic events especially in the setting of active disease. ${ }^{35,36}$ Thromboembolisms have been reported to arise in the arterial or venous system and in typical (deep extremity veins or pulmonary artery) or atypical (portal vein, retinal vein, central nervous system) locations. However, in a recent meta-analysis, the risk of VTE was particularly increased (by $96 \%$ ) in IBD patients versus the general population (relative risk 1.96; 95\% CI 1.67-2.30) while that of arterial thromboembolism was not (relative risk 1.15; 95\% CI 0.91-1.45). The magnitude of the risk of VTE was higher in studies including IBD patients in general versus those looking at hospitalized IBD patients. ${ }^{37}$ The incidence of VTE in UC is approximately $0.26 \%$ per year, and IBD activity is an independent risk factor for VTE development. ${ }^{36}$ The relative risk of VTE is increased during periods of disease flare (hazard ratio 8.4) and appears more prominent during ambulatory versus hospitalized periods. ${ }^{38}$ The prevalence of VTE appears to be greatest among IBD patients aged $\leq 40$ years and is higher among hospitalized UC versus CD patients. ${ }^{37,39}$ Mortality in IBD patients following VTE ranges from 8\%-25\%. Among hospitalized patients, the excess mortality associated with VTE is 2.1-fold higher for IBD patients than for non-IBD patients $(P<0.0001) .{ }^{39}$ The rate of VTE after UC-related surgery has been reported at $3.3 \%{ }^{40} \mathrm{~A}$ number of published guidelines recommend administration of prophylactically dosed anticoagulants, such as unfractionated or lowmolecular weight heparin, in hospitalized IBD patients. ${ }^{1,35}$ The rates of major and minor bleeding have been shown to be similar between hospitalized IBD patients receiving and not receiving VTE prophylaxis. ${ }^{41}$ The presence of rectal bleeding on admission is not a contraindication to prophylactic anticoagulation.

\section{Treatment options and goals of therapy}

Treatment for UC can become quite complex and requires an individualized and multidisciplinary approach. Collaboration among medical and surgical teams, nutritionists, psychiatrists, and social support services is important particularly in new-onset acute cases and those requiring complex decision making. Professional recommendations must then be synthesized and translated with a patient-centered, educational focus.

Patients with mild-to-moderate disease can usually be managed in the outpatient setting, while severe UC warrants inpatient care. In general, sequential therapy (Figure 1) based on disease severity and subsequent response is recommended 
for both new-onset cases and periods of flare. The goal of treatment in the acute setting is to induce remission and avoid complications. Ideally, the agent selected to achieve remission is carried forward to maintain disease control.

Table 2 provides a summary of 5-aminosalicylic acid (aminosalicylates [5-ASAs]) formulations used in the treatment of UC with details including indication, delivery site, acute disease characteristics, typical dosing (for active disease and maintenance of remission), and common adverse reactions. Table 3 outlines the major categories of immunosuppressant medications used in treating UC (corticosteroids, thiopurines, biologics, and calcineurin inhibitors) with details including indication, typical dosing, efficacy, and common side effects.

\section{Mild-to-moderate UC}

\section{5-ASAs, mesalamine, and topical corticosteroids}

Induction of remission for mild-to-moderate distal colitis can be achieved with topical mesalamine, oral 5-ASAs, or topical steroids with topical mesalamine as a superior first-line agent. ${ }^{1}$ Topical mesalamine has proven effective compared to placebo, ${ }^{42}$ oral 5-ASAs, ${ }^{43}$ and topical steroids. ${ }^{44}$ The superiority of topical mesalamine to oral 5-ASAs in achieving clinical improvement for mild-to-moderate distal colitis has been shown in a meta-analysis of controlled trials. ${ }^{45,46}$ Rectal administration is optimal for proctitis and left-sided colitis, while oral therapy is used for more extensive colonic disease.

Oral 5-ASA preparations have varying sites of delivery with the intent to deliver medication directly to sites of colonic inflammation with minimal systemic side effects. Sulfasalazine, balsalazide, and olsalazine are released in the colon; delayed-release mesalamine and multimatrix (MMX) mesalamine are active in the terminal ileum and colon (release at $\mathrm{pH} \geq 7$ ); granulated mesalamine works in the terminal ileum and colon (release at $\mathrm{pH} \geq 6$ ); and controlled-release mesalamine takes effect in the duodenum, jejunum, ileum, and colon. A systematic review examining mesalamine formulations and prodrugs demonstrated no differences in absorption and systemic exposure to 5 -ASA.${ }^{47}$ Effective doses of sulfasalazine range from 4-6 g/day in four divided doses, and balsalazide is dosed at $6.75 \mathrm{~g} /$ day in three divided doses. Olsalazine is dosed from 1.5-3 g/day in two divided doses; its benefit, however, has not been established in the setting of active UC perhaps due to the confounding potential side effect of diarrhea. ${ }^{1}$ Effective doses of oral mesalamine range from 4-6 g/day in three divided doses. The MMX mesalamine formulation provides comparable results with a convenient dosing regimen of 2.4-4.8 g once daily. ${ }^{48,49}$ Prolonged-release mesalazine dosed at $4 \mathrm{~g}$ once daily appears to be as well-tolerated and effective for inducing remission in mild-to-moderately active UC compared to a $2 \mathrm{~g}$ twice daily dosing regimen. ${ }^{50}$ A multicenter, randomized, double-blind, active-control study conducted over 6 weeks compared delayed-release mesalamine $4.8 \mathrm{~g} /$ day with $2.4 \mathrm{~g}$ /day for moderately active UC; treatment success (overall improvement) using the Physician's Global Assessment (based on parameters of rectal bleeding, stool frequency, and sigmoidoscopy with no worsening in any individual clinical assessment) was seen in $70 \%$ of patients receiving $4.8 \mathrm{~g}$ /day versus $66 \%$ in those receiving $2.4 \mathrm{~g} /$ day. Higher doses of mesalamine

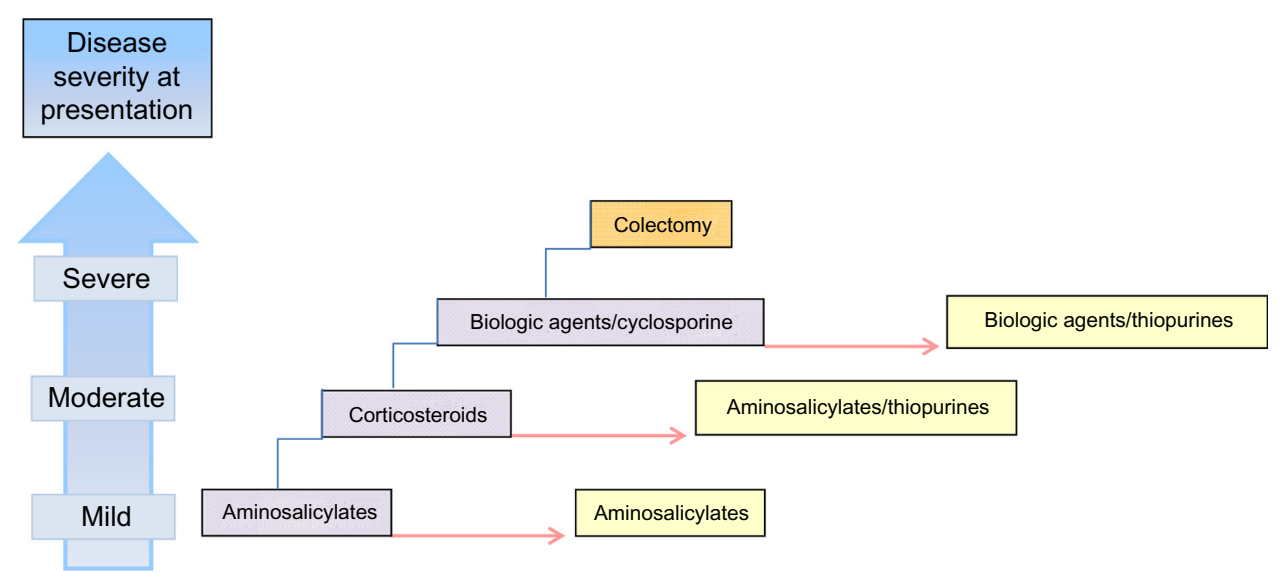

Figure I Sequential therapy in the treatment of ulcerative colitis based on disease severity at presentation (blue boxes, left). Initial treatment options based on disease category are shown (purple boxes). Therapy is escalated based on severity at presentation or failure to respond to prior step. Red arrow signifies time, and maintenance options are shown (yellow boxes). Colectomy (orange box) is considered in fulminant or recalcitrant disease. 


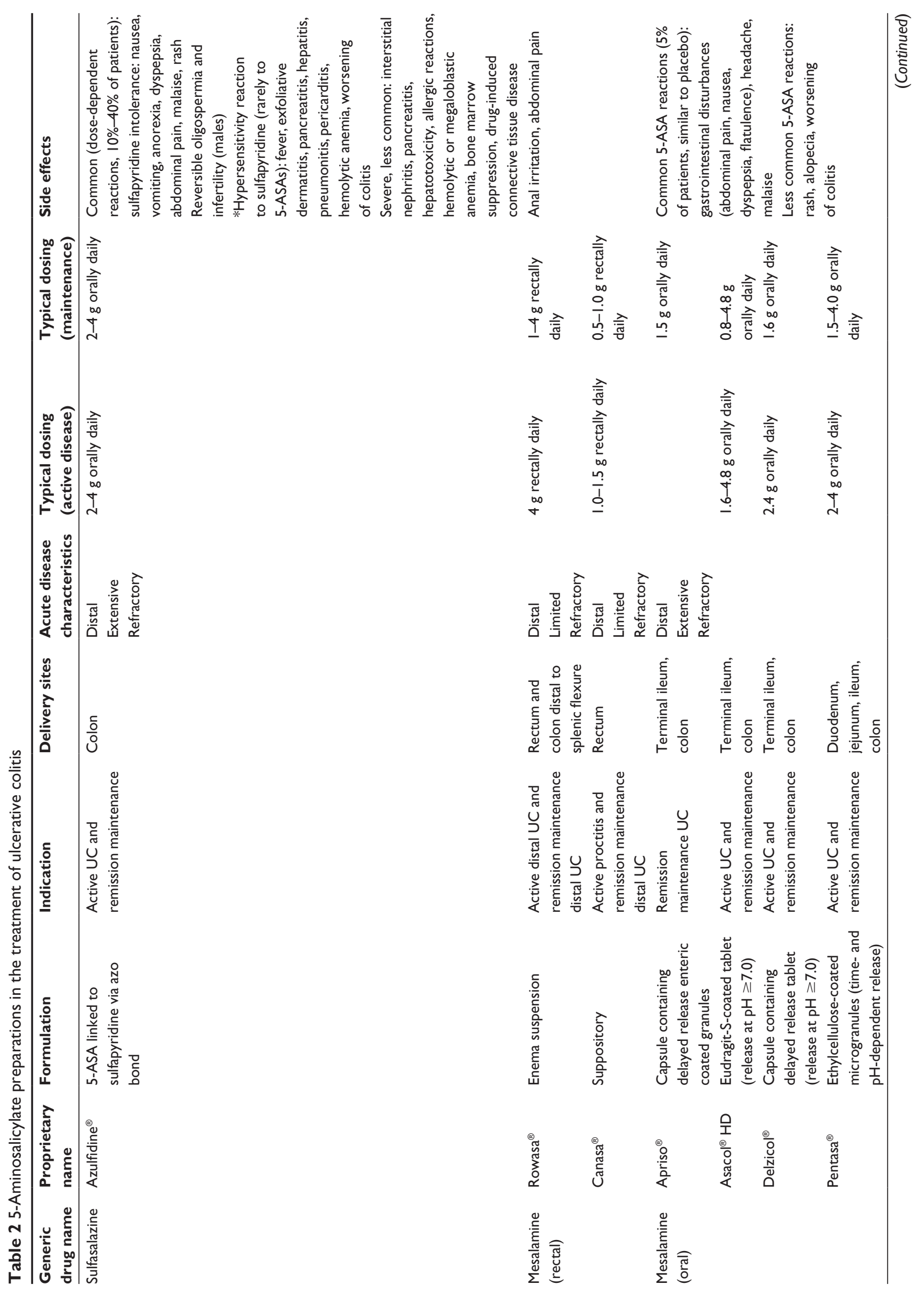




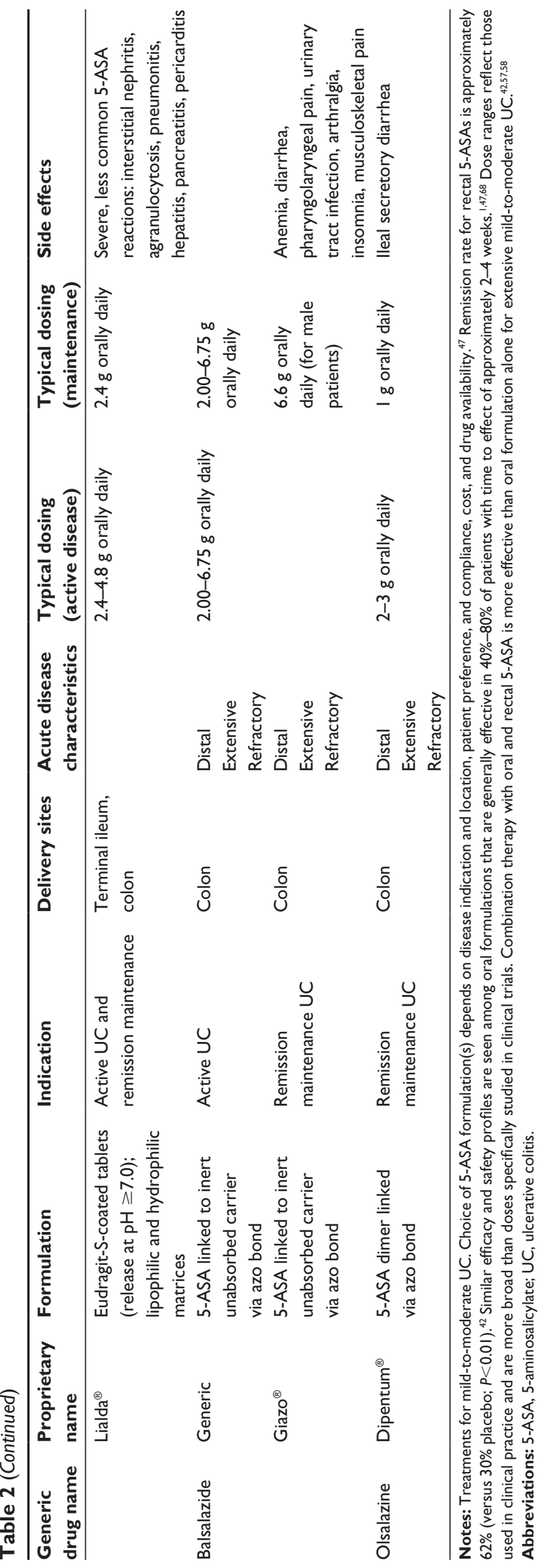

appeared to be effective and well-tolerated. At 6 weeks, patients receiving $4.8 \mathrm{~g}$ /day achieved significantly higher clinical remission rates versus those receiving $2.4 \mathrm{~g}$ /day ( $43 \%$ versus $35 \%$, respectively; $P=0.04$ ). Both dosing regimens had similar reported tolerability and side effects. ${ }^{51}$

Historically, early registration trials used four times daily or three times daily 5-ASA dosing to mirror the regimen used for sulfasalazine. However, post-marketing data suggests that less frequent dosing of agents for induction and maintenance is possible, ${ }^{48,52-55}$ and newer agents are indicated for once daily dosing. ${ }^{48,56}$

Oral 5-ASAs are effective in approximately $40 \%-80 \%$ of patients and demonstrate effect in about 2-4 weeks, ${ }^{1}$ although longer treatment induction courses may be required. Patients who fail to respond to the first 8 weeks of mesalamine therapy may achieve clinical and endoscopic remission with an 8-week, high-dose MMX mesalamine extension before being considered for step-up therapy with steroids or immunosuppressants. ${ }^{49}$ Oral mesalamine ( 2.4 and 4 g/day) combined with mesalamine enema achieves a more rapid response and more effective clinical improvement versus either agent alone. ${ }^{57,58}$

Topical agents as an alternative or supplement to oral 5-ASAs include mesalamine suppositories or enemas and hydrocortisone foam or enemas. In general, suppositories reach the upper rectum (10 $\mathrm{cm}$ above the anal verge), while enemas can extend as far as the splenic flexure and distal transverse colon. Foams can be effective for disease involving the rectum and distal sigmoid $(15-20 \mathrm{~cm}$ above the anal verge) but do not reach as far as enemas. ${ }^{1,46}$ Mesalamine suppositories or enemas may be effective in inducing remission for patients unresponsive to oral 5-ASAs or topical steroids particularly for patients with proctitis and distal symptoms. ${ }^{59}$ Mesalamine suppositories can be dosed at $500 \mathrm{mg}$ twice daily or $1 \mathrm{~g}$ once daily; doses of 1 g administered nightly are considered optimal. ${ }^{1,60}$ The high efficacy and low side effect profile of rectal 5-ASA suppositories makes this formulation the treatment of choice for ulcerative proctitis. ${ }^{46}$ Mesalamine enemas in doses of 1-4 g/day can cover more proximal disease to the splenic flexure and can induce and maintain remission in distal colitis. ${ }^{61,62}$ Mesalazine foam (not available in the US) and enemas are equally effective for the treatment of active proctitis, proctosigmoiditis, and left-sided colitis; ${ }^{63}$ the foam modality is often better tolerated with better rectal retention. ${ }^{1}$

Topical corticosteroids are effective in the treatment of acute distal colitis and are available in the US as a 
hydrocortisone enema $(100 \mathrm{mg})$ or $10 \%$ hydrocortisone foam. Patients intolerant of or unresponsive to rectal mesalamine after 2 weeks can be switched to hydrocortisone formulations. Other effective rectal corticosteroids include beclomethasone and budesonide. Beclomethasone dipropionate and mesalamine foam and enema have similar efficacy and tolerability in mild active distal colitis. ${ }^{64}$ Budesonide is a second-generation corticosteroid with low systemic bioavailability $(10 \%-15 \%)$ due to first-pass hepatic metabolism and has resultant modest effects on the hypothalamic-pituitary-adrenal axis. As a compounded enema, budesonide is safe and effective for treating active distal UC and proctitis with a lowest effective dose of $2 \mathrm{mg} / 100 \mathrm{~mL} .{ }^{65}$ As an oral formulation, budesonide is absorbed in the gastrointestinal tract more proximal to the colon; MMX technology allows extension of release into the colon. Oral budesonide MMX at a dose of $9 \mathrm{mg}$ / day has recently proven more effective than placebo in inducing endoscopic and clinical remission in mild-tomoderate UC. ${ }^{66,67}$

Topical therapy generally provides a more rapid response with less frequent dosing and less systemic side effects versus oral therapy. Agents are determined by both patient preference and proximal disease extent. ${ }^{1}$ Although they can help significantly with distal disease, topical therapies may pose difficulty or discomfort for patients secondary to the sense of tenesmus. Administration can be inconvenient, especially while outside of the home or in social situations. Patients can also experience anal leakage that could be uncomfortable and embarrassing; foam modalities may be easier to tolerate in this regard.

Sulfasalazine is a prodrug composed of sulfapyridine bonded to active mesalamine, and up to one-third of treated patients experience side effects thought to be attributed to the sulfur moiety. ${ }^{68}$ Common reactions include headache, indigestion, nausea, vomiting, and abdominal pain. More severe but less frequent effects include pancreatitis, hepatotoxicity, allergic reactions, bone marrow suppression, drug-induced connective tissue disease, hemolytic or megaloblastic anemia, and interstitial nephritis. ${ }^{1}$ Reversible sperm abnormalities (count/morphology/motility) can be seen related to the sulfapyridine component but are not seen with mesalamine products. 5-ASA products containing only the active ingredient were engineered with the intent to reduce the aforementioned side effects. Approximately $80 \%$ of patients intolerant to sulfasalazine are able to handle olsalazine, balsalazide, and mesalamine; some allergic reactions formerly attributed to the sulfur component of sulfasalazine have been documented with the newer 5-ASA preparations. ${ }^{69}$

\section{Moderate-to-severe UC Oral and IV corticosteroids}

Patients who are refractory to rectally administered 5-ASAs and/or corticosteroids or oral 5-ASAs at maximal doses may require systemic corticosteroids. ${ }^{46} \mathrm{~A}$ typical dosing regimen is oral prednisone 40-60 mg/day (or equivalent) for 1-2 weeks until clinical response is established followed by steroid taper by $5-10 \mathrm{mg} /$ week depending on disease severity and rapidity of response at onset of therapy. Once at a $20 \mathrm{mg}$ dose, tapering of a patient's dose customarily proceeds at $2.5 \mathrm{mg} /$ week. A double-blind, non-placebo-controlled outpatient trial of 58 patients with active mild UC examining optimal oral prednisone dosing at 20,40 , and $60 \mathrm{mg}$ daily found that 40 or $60 \mathrm{mg}$ daily dosing achieved greater symptomatic improvement with two times the remission rate versus $20 \mathrm{mg}$ daily over 2 weeks. Additionally, patients in the $40 \mathrm{mg}$ arm experienced fewer side effects than those in the $60 \mathrm{mg}$ arm. Thus, oral prednisone starting at $40 \mathrm{mg}$ daily has been considered optimal. ${ }^{70}$ Oral and rectal 5-ASA agents should generally be continued through the corticosteroid course with the intent of maintaining remission at completion of the taper. ${ }^{1,46}$

Severe UC or failure of response to oral steroids requires consideration for hospitalization and IV steroid therapy with a recommended dosing regimen of methylprednisolone (40 mg to $1 \mathrm{mg} / \mathrm{kg}$ ) as a single IV bolus. Administering IV methylprednisolone at doses $>60 \mathrm{mg}$ /day is not supported by current literature. ${ }^{71}$ Randomized controlled trials of IV corticosteroids for severe UC are lacking. Sixty percent of patients hospitalized for severe UC will achieve clinical remission within 5-7 days of treatment initiation. ${ }^{32}$ A systematic review of response to corticosteroids in severe UC incorporating 32 cohort and controlled studies from 1974-2006 (1,991 patients) revealed an average colectomy rate of about $27 \%$ and death rate of $1 \% .^{71}$ Stool frequency, disease extent, temperature, heart rate, C-reactive protein, albumin, and radiologic assessments were consistently reported variables involved in treatment failures. ${ }^{71}$

Steroids carry a significant side effect profile and can distort metabolic activity in a multitude of organ systems. Such adverse effects include diabetes, cushingoid features, glaucoma, cataracts, and psychiatric instability. ${ }^{1}$ The risk for opportunistic infections is increased about threefold in IBD patients exposed to steroids with a higher relative risk in patients aged over 50 years; when steroids are combined with other immunosuppressants, this risk elevates 


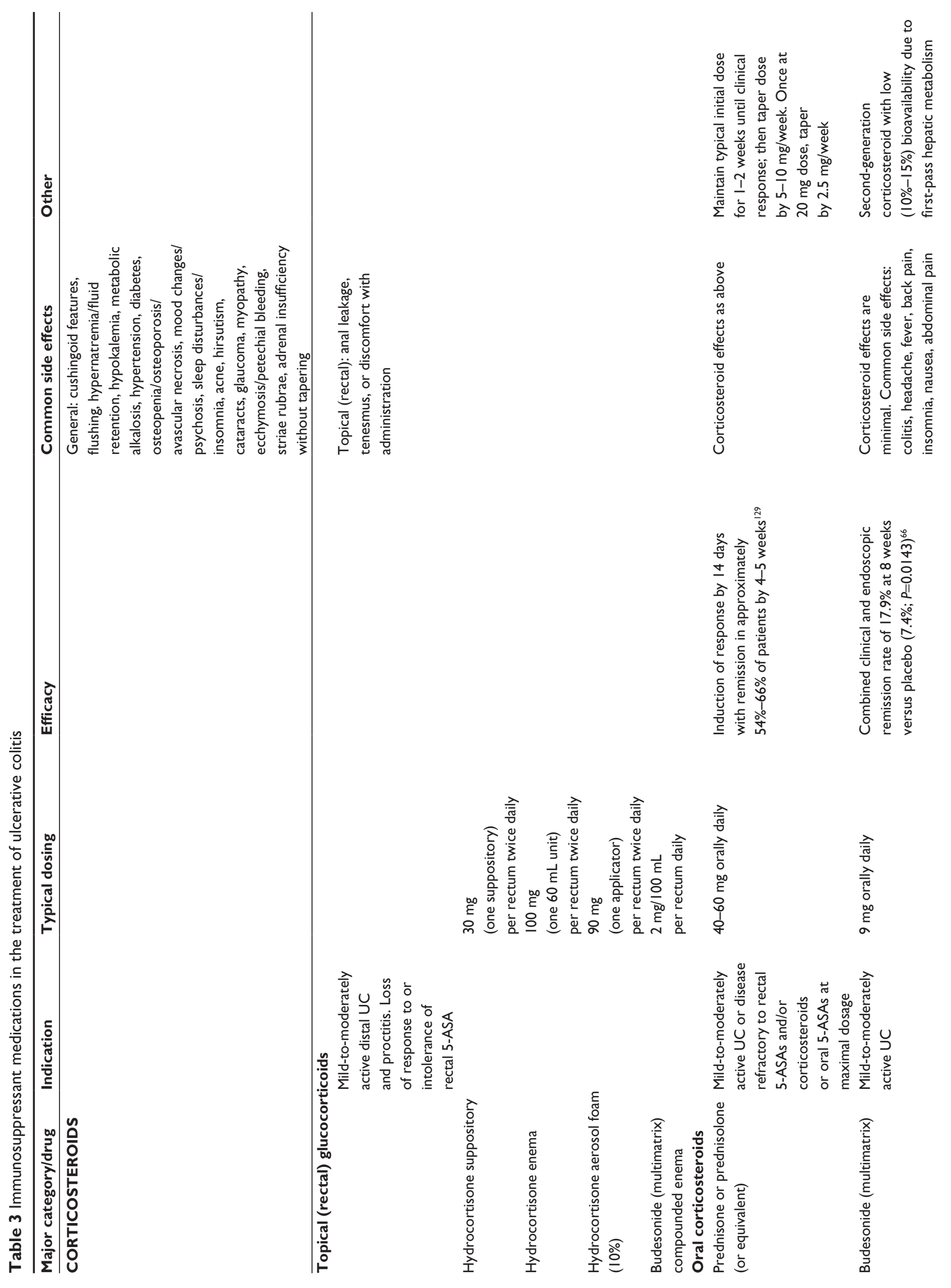




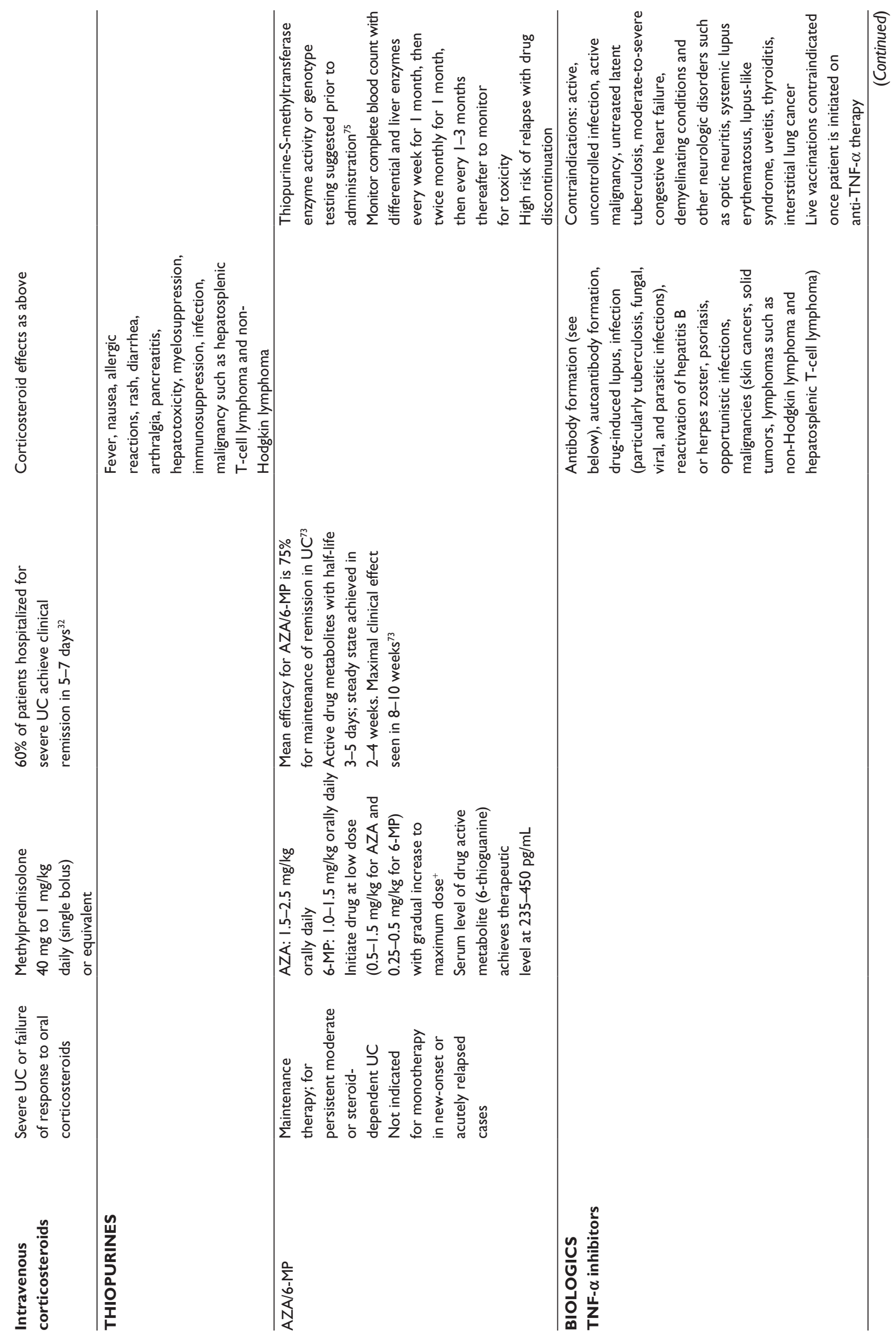




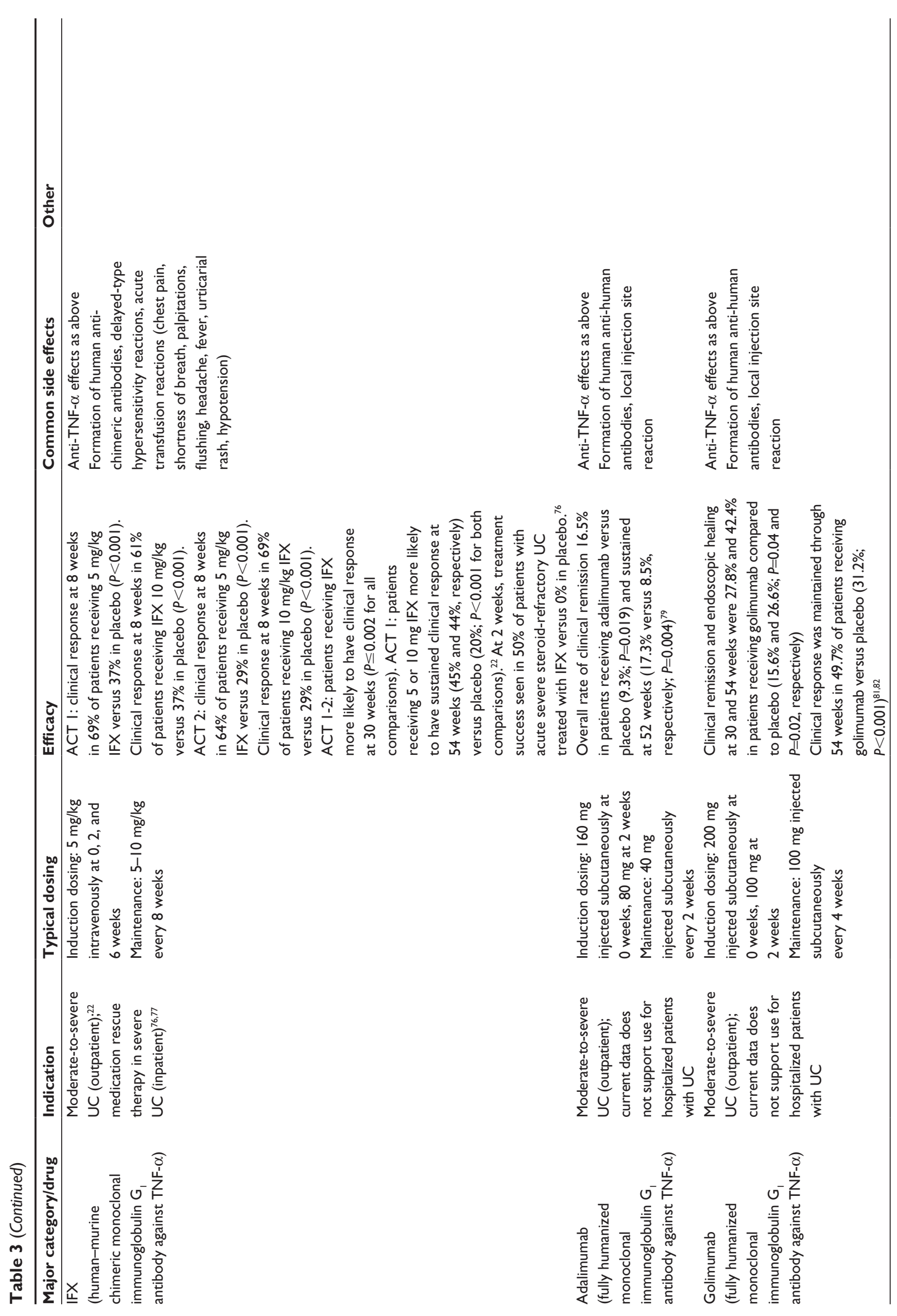



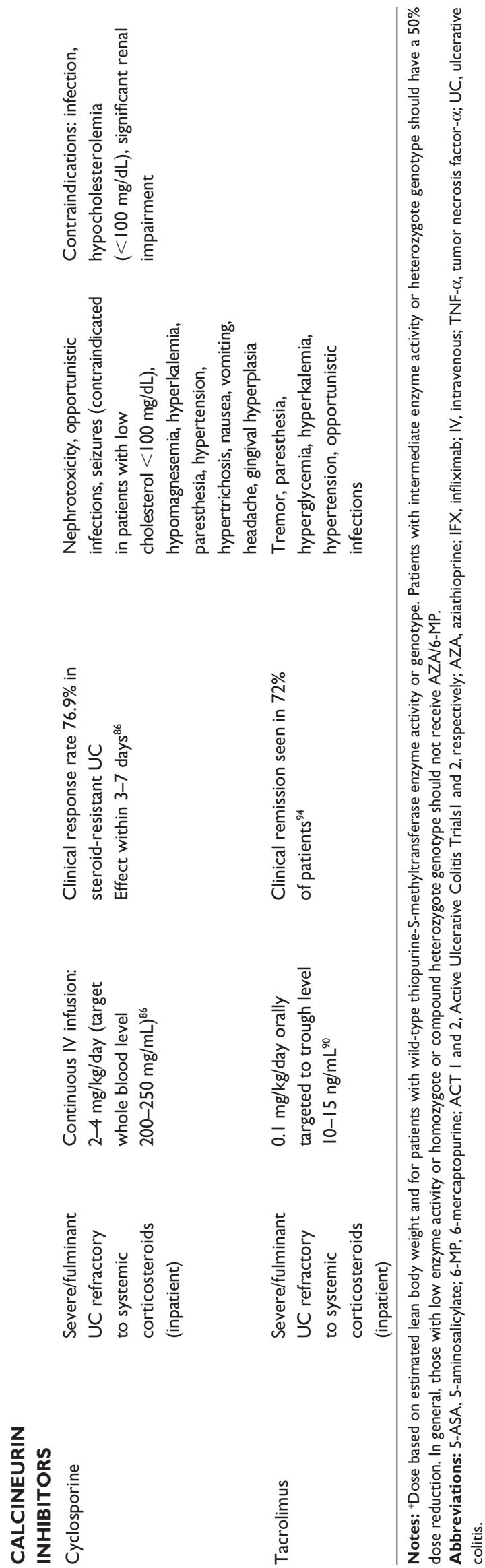

substantially. ${ }^{72}$ Other steroid side effects include impaired wound healing, hyperlipidemia, metabolic bone disease, hypernatremia/fluid retention, hypokalemia, metabolic alkalosis, steroid myopathy, and accelerated atherosclerosis. Adrenal insufficiency can develop and requires consideration when tapering. ${ }^{1}$ Glucocorticoid effects of budesonide MMX are minimal and appear to be similar to placebo; most commonly reported effects include colitis, headache, and pyrexia. Drug-related adverse events are low, and serious side effects leading to treatment abortion are infrequent. ${ }^{66}$

\section{Thiopurines}

AZA and 6-mercaptopurine have limited utility in the acute setting. The active drug metabolites have a half-life of approximately $3-5$ days. A steady state can be achieved after about 2-4 weeks, but maximal clinical effect can take up to $8-10$ weeks. ${ }^{73}$ A prospective, investigator-blinded, randomized controlled trial comparing AZA and 5-ASA for induction of remission in steroid-dependent $U C$ patients found that AZA is significantly more effective than 5-ASA in achieving clinical and endoscopic remission with discontinuation of steroids at 6 months. ${ }^{74}$ Although not indicated to induce remission in the acute setting, thiopurines can be used as an adjunct to infliximab (IFX) therapy or as a maintenance medication after treatment with tacrolimus or CsA. Thiopurines offer a steroid-sparing maintenance therapy and can be considered for patients with persistent moderate disease who are unresponsive to/cannot be weaned from steroids and who do not meet the need for IV alternatives. ${ }^{1}$ Thiopurines are not well-studied in the setting of limited distal disease. Thiopurine methyltransferase enzyme activity should be assessed prior to inception of the therapy to determine recommended starting dose and potential for early severe side effects. ${ }^{75}$ Adverse effects of thiopurines include fever, rash, nausea, diarrhea, arthralgia, thrombocytopenia, leukopenia, infection, pancreatitis, hepatitis, non-Hodgkin lymphoma, and hepatosplenic T-cell lymphoma among others.

\section{Biologic agents: tumor necrosis factor- $\alpha$ (TNF- $\alpha$ ) inhibitor therapy IFX}

IFX is a chimeric human-murine monoclonal immunoglobulin $\mathrm{G}_{1}$ antibody against TNF- $\alpha$. It is indicated for moderateto-severely active UC in the outpatient setting. ${ }^{22}$ IFX has also been shown in two placebo-controlled trials to be effective in hospitalized patients with severe active UC unresponsive to conventional therapy. ${ }^{76,77}$ Induction dosing for IFX is $5 \mathrm{mg} / \mathrm{kg}$ 
IV at 0,2 , and 6 weeks followed by maintenance dosing at $5-10 \mathrm{mg} / \mathrm{kg}$ every 8 weeks. Two randomized double-blind, placebo-controlled trials (Active UC Trials 1 and 2 [ACT 1 and ACT 2, respectively]) evaluating the efficacy of IFX versus placebo for induction and maintenance therapy in moderate-to-severe UC with failed medical therapy have demonstrated sustained clinical response and remission at 8 and 30 weeks in both trials and at 54 weeks in ACT 1; patients receiving IFX were significantly more likely to have endoscopic mucosal healing at these landmarks. ${ }^{22}$ At baseline, $61.0 \%$ and $51.1 \%$ were receiving steroids in ACT 1 and 2, respectively, with a median daily dose of $20 \mathrm{mg} /$ day; a higher proportion of patients in the IFX versus placebo group achieved clinical remission and discontinued steroids at 30 weeks (ACT 1 and 2) and at 54 weeks (ACT 1). Adverse events were similar in placebo compared to IFX groups.

IFX is contraindicated for use in patients with active uncontrolled infection, moderate-to-severe congestive heart failure, untreated latent tuberculosis, preexisting demyelinating conditions, optic neuritis, and active malignancies. Acute transfusion reactions (characterized by chest pain, shortness of breath, palpitations, flushing, headache, fever, and sometimes urticarial rash or hypotension) and delayed-type sensitivity reactions may occur with administration. Other adverse events include the development of opportunistic infections, non-Hodgkin lymphoma, hepatosplenic T-cell lymphoma, and skin cancers among others. ${ }^{1}$

\section{Adalimumab (ADA)}

ADA is a fully humanized monoclonal antibody against TNF- $\alpha$. Current data does not support use of ADA for hospitalized patients with UC, and the comparative efficacy between IFX and ADA has not been reported. A randomized, double-blind, placebo-controlled trial was conducted to assess the efficacy of ADA for induction and maintenance of moderate-to-severe UC unresponsive to conventional medical therapy. Clinical remission was seen after 8 weeks of therapy. ${ }^{78}$ Clinical response at 8 weeks was seen in $50 \%$ of patients receiving $160 \mathrm{mg}$ at week 0 and $80 \mathrm{mg}$ at week 2 versus $35 \%$ in the placebo group $(P<0.001)$. Standard induction dosing is $160 \mathrm{mg}$ subcutaneously at week 0 and $80 \mathrm{mg}$ subcutaneously at week 2 followed by $40 \mathrm{mg}$ subcutaneously every 2 weeks, starting at week $4 .{ }^{79}$ Recent data supports that adding ADA to standard UC therapy significantly reduces risks of all-cause, UC-related, and UC- or drug-related hospitalizations (40\%, 50\%, and 47\%, respectively; $P<0.05$ ) as observed over the first 8 weeks of therapy in patients with moderate-to-severe UC versus placebo; furthermore, decreased incidence rates for all-cause $(0.18$ versus 0.26 ; $P=0.03)$, UC-related ( 0.12 versus $0.22 ; P=0.02)$, and UC- or drug-related $(0.024$ versus $0.24 ; P=0.005)$ hospitalizations were observed during 1 year of ADA therapy versus placebo. Rates of colectomy did not differ between the two groups. ${ }^{80}$ Adverse events of ADA are similar to those of IFX.

\section{Golimumab}

Golimumab is a fully humanized monoclonal antibody against TNF- $\alpha$ used to induce clinical response, remission, and mucosal healing in moderate-to-severe UC unresponsive to conventional medical therapy. ${ }^{81}$ A Phase III double-blind trial of patients with moderate-to-severe UC responsive to golimumab induction (Program of UC Research Studies Utilizing an Investigational Treatment) demonstrated that golimumab injected at 50 or $100 \mathrm{mg}$ subcutaneously once monthly maintained clinical response through 54 weeks in $47 \%$ of patients dosed with $50 \mathrm{mg}$ golimumab, $49.7 \%$ of patients dosed with $100 \mathrm{mg}$ golimumab, and $31.2 \%$ of patients dosed with placebo ( $P=0.10$ and $P<0.0001$, respectively). Patients receiving $100 \mathrm{mg}$ golimumab attained higher rates of clinical remission and mucosal healing compared to $50 \mathrm{mg}$ golimumab (23.3\% and 41.7\%, respectively) and to placebo (27.8\% and $42.4 \%$ versus $15.6 \%$ and $26.6 \% ; P=0.004$ and $P=0.002$, respectively). Patients receiving a $100 \mathrm{mg}$ dose had both clinical remission and endoscopic healing at 30 and 54 weeks. ${ }^{82}$ The safety profile is similar to that reported for other anti-TNF agents. Golimumab recently received Food and Drug Administration approval for UC in the US and is indicated for moderate-to-severe UC for outpatients.

\section{Severe UC}

Acute severe colitis afflicts approximately $25 \%$ of patients with $\mathrm{UC}^{83}$ and the mainstay of therapy is IV corticosteroids. Patients who are fulminant or refractory to systemic glucocorticoids require salvage medical therapy with CsA or IFX (see section above), or colectomy. Severity of disease at admission is related to outcome. Timely consideration of surgery is crucial in this subset of patients experiencing a potentially life-threatening situation that carries a high risk of colectomy. Approximately $30 \%-40 \%$ of patients are refractory to steroids and require emergent colectomy as a life-saving surgery. ${ }^{19}$

\section{Clinical prediction of steroid responsiveness}

Clinical predictions have been established in an attempt to determine which patients will respond to IV corticosteroid 
therapy through several investigations. In one study of 51 inpatients undergoing intensive therapy for severe UC, predictive factors of colectomy on same hospital admission included stool frequency of more than eight per day or three to eight stools with C-reactive protein levels ( $>45 \mathrm{mg} / \mathrm{L}$ ) assessed on day 3 of hospitalization with $85 \%$ success. Incomplete responders having more than scant bloody stools per day after 1 week of treatment had a $60 \%$ chance of continuous symptoms and a $40 \%$ chance of colectomy in the months following hospitalization. ${ }^{84}$ The Ho Index is an updated clinical prediction scoring system formulated through a retrospective analysis of 167 consecutive patients at one medical center with the purpose of identifying severe UC patients who may be unresponsive to IV steroid therapy at an early stage. Patients are assessed on day 3 of IV corticosteroid administration with points assigned for stool frequency, albumin, and colonic dilation; a total score of more than three has an $80 \%$ positive predictive value for colectomy. Additionally, the Ho Index appears to predict response to CsA and colectomy avoidance after failure of corticosteroid treatment over 5 days. ${ }^{85}$ The Ho Index has not been prospectively validated. Although clinical prediction scoring is not a substitute for clinical judgment, it may aid in organizing realistic plans and anticipating need for potential surgery.

\section{CsA}

CsA, a calcineurin inhibitor, is a rapidly-acting immunosuppressant effective in treating severe refractory UC. IV CsA appeared to be safe and effective for treating moderately severe steroid-refractory UC in a small prospective open-label study. ${ }^{33}$ Doses of $3.7 \pm 0.5 \mathrm{mg} / \mathrm{kg}$ IV were given until response was achieved followed by oral dosing for 3.5 \pm 2.6 months. Clinical response was seen in almost $77 \%$ of patients within $5.7 \pm 2.8$ days. Relapse rate was $60 \%$ at approximate 28-month follow-up; five of six treatment failures underwent proctocolectomy. The probability of surgical avoidance was $52 \%$ at 78 months, and the sole factor associated with this was concomitant AZA therapy $(P=0.007) .{ }^{86} \mathrm{~A}$ retrospective analysis of 24 patients who received IV CsA ( $4 \mathrm{mg} / \mathrm{kg} /$ day for an average of 6.63 days) for severe steroid-refractory UC followed by oral CsA dosed for 3 months showed that colectomy was avoided in approximately $79 \%$ during the early stages of treatment. Three patients required surgery over time (average 38 months; range 12-62 months), but $67 \%$ remained colectomy-free overall. One patient required colectomy in the acute setting due to convulsions with IV CsA administration. ${ }^{87}$
CsA is given as a continuous IV infusion of $2 \mathrm{mg} / \mathrm{kg}$ over 24 hours (target whole-blood level 200-250 mg/mL). A typical response is achieved within 1 week, and lack of response warrants colectomy. Patients showing a response to IV CsA should be transitioned to oral CsA beginning with doubled dose of the IV formulation (goal whole-blood trough level $200-250 \mathrm{mg} / \mathrm{mL}$ ). Oral CsA should be bridged with AZA or 6-mercaptopurine for 2-3 months prior to consideration of CsA taper. Steroid-dependent chronic active UC patients placed on oral CsA $(5 \mathrm{mg} / \mathrm{kg} /$ day titrated to reach whole-blood therapeutic range $60-240 \mathrm{mg} / \mathrm{mL}$ ) with steroid tapering over 3 months showed an $89 \%$ initial response rate and about $56 \%$ remission rate in a very small retrospective study. ${ }^{88}$ Larger studies are needed to validate this concept of oral CsA as a steroid-sparing maintenance medication in chronic UC. Up to one-third of patients with severe UC are unresponsive to CsA and must be considered for alternative therapy. ${ }^{86,89}$

Contraindications to use of CsA include infection, hypocholesterolemia (seizure risk), and significant renal impairment. Reported major toxicities of CsA dosed at $2-4 \mathrm{mg} / \mathrm{kg}$ for severe steroid-refractory UC include nephrotoxicity (24\%), infection (20\%), seizure (4\%), and death $(1 \%-2 \%){ }^{32}$ Other adverse effects include paresthesia (51\%), hypertension (39\%), hypertrichosis (27\%), hypomagnesemia (20\%), hyperkalemia (13\%), nausea, vomiting, headache, tremors, and gingival hyperplasia. ${ }^{87}$

\section{Tacrolimus}

Tacrolimus is a calcineurin inhibitor that can be considered in cases of severe steroid-refractory UC. A 2-week double-blind, placebo-controlled study of oral tacrolimus therapy performed in hospitalized patients with steroidrefractory moderate-to-severe UC revealed that treated patients whose tacrolimus drug trough level was targeted at $10-15 \mathrm{ng} / \mathrm{mL}$ were more likely than placebo to achieve clinical response, although they were unable to achieve complete remission. ${ }^{90}$ Some open-label series have also been published showing efficacy and relative safety of tacrolimus as a rapid-acting option in treating severe $\mathrm{UC} \cdot{ }^{11-93} \mathrm{~A}$ recent retrospective three-center study of 130 patients with moderate-to-severely active steroid-refractory UC analyzed patients within 3 months of initiation of tacrolimus. In most cases, an oral dose was given at $0.1 \mathrm{mg} / \mathrm{kg} /$ day; in a minority of patients, an IV dose was given at $0.01 \mathrm{mg} / \mathrm{kg} /$ day with a fast transition to oral formulation. Daily tacrolimus doses were adjusted at the discretion of the treating physician based on clinical requirements. Steroids were tapered 
individually and, in some cases of prior refractoriness, were not even started; extant thiopurines were continued. Most patients (59\%) in the series had pancolitis. At 12 weeks, the median Lichtiger score decreased from 13 at baseline to three. Clinical remission was achieved in $72 \%$, and 18 patients required colectomy within the 3 -month period after tacrolimus treatment. Overall adverse effects due to tacrolimus were seen in $53 \%$ of patients, leading to medication discontinuation in two cases (due to drug intolerance and increased serum creatinine). The most common reported side effects in this study were tremor/ paresthesia (38\%) and hyperglycemia (10\%). Concomitant thiopurine use significantly increased remission $(P=0.002)$ and favored colectomy sparing in the short-term. ${ }^{94}$ Side effects of tacrolimus include acute kidney injury, tremor, paresthesia, hypertension, hyperkalemia, and opportunistic infections. ${ }^{92}$ Overall, insufficient available data limit the ability to guide optimal initial and maintenance dosing and follow-up care.

\section{Medical rescue therapy considerations and failure of response}

Risks and benefits of medical rescue therapies should be weighed with consideration for maintenance therapy options; for example, a potential benefit of IFX is that it can be continued in patients who respond to initial therapy. Concomitant thiopurine administration should be considered in patients receiving CsA or IFX who are appropriate candidates, although this further compounds immunosuppression.

Patients failing to show a response to rescue therapy within 5-7 days should undergo colectomy. Switching from one to another rescue therapy can achieve remission in 30\%-40\% of cases. However, in the setting of intense immunosuppression, serious adverse events including infections (16\%-20\% of patients) as well as death have been reported. ${ }^{32,95}$

\section{Surgery}

Approximately $27 \%$ of patients with acute severe UC undergo colectomy, ${ }^{32}$ and extensive colitis is a risk factor for surgery. The cumulative probability of colectomy in relation to time of diagnosis has been reported as $13.1 \%$ at 5 years $(95 \%$ CI 9.4\%-16.6\%), $18.9 \%$ at 10 years (95\% CI 14.4\%-23.3\%), and $25.4 \%$ at 20 years (95\% CI 19.8\%-30.8\%). The shortterm colectomy rate in cases of severe hospitalized UC has been constant around $27 \%$ for multiple years. ${ }^{10,32}$ In one study, patients requiring hospitalization for medical management of UC were five times more likely to require colectomy after adjustments for other factors; need for IFX therapy was a second independent predictor of colectomy. ${ }^{96}$ Patients $>65$ years of age have an increased rate of early colectomy (within 3 months of diagnosis). ${ }^{10}$

The importance of early surgical involvement cannot be overemphasized. Emergent surgical indications include severe disease or fulminant colitis unresponsive to medical therapy, perforation, toxic megacolon, and refractory hemorrhage. Perforation in patients with UC can occur without typical signs of peritonitis and in the absence of colonic dilation. Identifying patients who require early surgery is important because delayed surgical intervention can lead to poor shortterm outcomes ${ }^{97}$ and measureable longer-term mortality. ${ }^{9}$ In a retrospective review of patients undergoing surgery for fulminant UC, those who developed postoperative complications underwent colectomy significantly later (3.6 versus 7.4 days; $P=0.01)$ than those without such complications. Specifically, patients who underwent $>72$ hours of preoperative medical therapy had a significantly higher risk of developing a major postoperative complication. Major complications occurred in $27 \%$ of patients, and those most frequently reported included infection (superficial and organ-space surgical site infections) and small bowel obstruction. Sustaining postoperative complications, however, did not appear to influence eventual pursuit of ileal pouch-anal anastomosis (IPAA; 68\% versus $77 \% ; P=0.5) .^{98}$

Emergent colectomy bears a 30-day mortality rate of about $5 \%$ while that of elective surgery is less. ${ }^{9,97}$ The goal of emergency surgery is to treat fulminant colitis, restore patient health, and subsequently allow for systemic recovery with nutrition optimization in preparation for a future procedure. Emergent UC surgery typically requires a three-stage IPAA approach beginning with total or subtotal abdominal colectomy and diverting end ileostomy that leaves behind a rectal or rectosigmoid stump as a Hartmann's pouch. This procedure eliminates most of the diseased colon in a relatively straightforward fashion, avoiding pelvic dissection and intestinal anastomosis. ${ }^{99}$ Disease in the remaining rectum after emergent surgery is generally inconsequential; a majority of patients can discontinue previous colitis medications. ${ }^{100}$ After sufficient recovery, patients can then undergo a future restorative surgery (described below) or a completion proctectomy with end ileostomy.

Restorative proctocolectomy with IPAA is currently the standard of care for patients undergoing elective surgery for UC. The procedure is most often completed as a twostage process and can be performed with an open, robotic, or laparoscopic approach. The first stage is a total procto- 
colectomy with creation of IPAA and temporary diverting loop ileostomy. The ileostomy serves to avert fecal material, allowing for healing of the pouch-anal anastomosis. The second surgical stage, undertaken after a few months, involves reversing the loop ileostomy and restoring intestinal continuity. The two-stage approach may reduce the risks of abdominal leakage and sepsis. ${ }^{99}$ Modifications of this two-stage system include excluding diversion at initial surgery (one-stage procedure) and performing a subtotal colectomy in a separate procedure prior to creating a diverting IPAA (three-stage procedure). The three-stage system has been used for patients with severely active disease, those on high-dose or long-term steroid therapy, or in cases without a clear diagnosis (namely UC versus CD). This procedure carries an overall morbidity rate of $19 \%-27 \%$ and a mortality rate of $0.2 \%-0.4 \%$. Additionally, postoperative QOL approaches that of the general population. ${ }^{100}$ In a single-institution retrospective study comparing two-stage and three-stage IPAA procedures, the decision to perform a three-stage procedure was affected by emergent indication $(P<0.001)$ and hemodynamic instability $(P=0.04)$ but was not influenced by such factors as use of steroids or anti-TNF agents. Interestingly, two-stage IPAA procedures did not change the risk of anastomotic leakage when accounting for all operations (odds ratio 1.09; $P=0.94$ ). An increased risk of perioperative complications in patients undergoing twostage versus three-stage procedures appeared to be affected by surgeon experience in IBD $(P=0.02)$ versus creation of an IPAA at initial operation $(P=0.55) .{ }^{101}$

Although curative in UC, surgery is associated with potential risks and complications. These include infection, hemorrhage, small bowel obstruction, intra-abdominal or pelvic abscess/sepsis, anastomotic strictures, fistulas, functional pouch issues including leakage and incontinence, pouchitis, cuffitis, reduced female fertility, male impotence and/or reverse ejaculation, sexual dysfunction, and surgical revision or pouch excision.

\section{Antibiotics}

Antibiotics have not proven to be of therapeutic benefit in the management of acute UC. If a documented or suspected coexisting infection exists, antibiotics should be tailored to the inciting organism. A double-blind, placebo-controlled trial of oral tobramycin as an adjunct to steroid therapy improved short-term outcomes in acute relapsing $\mathrm{UC}^{102}$ but showed no maintenance benefit. ${ }^{103}$ One study showed that a 6-month course of oral ciprofloxacin in addition to conventional treatment with mesalamine and prednisone decreased the rate of treatment failure, ${ }^{104}$ but this result was not sustained in other trials. In a prospective randomized controlled trial, oral ciprofloxacin as an adjunct to corticosteroids did not influence remission in patients with mild or moderately active UC. ${ }^{105}$ Similarly, IV ciprofloxacin did not augment corticosteroid therapy in the treatment of acute, severe UC. ${ }^{106}$ In a prospective double-blind trial, the addition of oral vancomycin (versus placebo) to conventional medical therapy for exacerbated colitis showed no significant overall difference in outcomes. ${ }^{107}$ In cases of severe UC, routine use of IV tobramycin and metronidazole ${ }^{108}$ or IV metronidazole ${ }^{109}$ in addition to corticosteroids is not supported. However, intensive IV regimens, generally including broad-spectrum antibiotics, have been advocated for patients with evidence of severe toxicity or worsening symptoms despite optimal medical therapy. Such regimens have provided the benefits of inducing clinical remission or determining need for emergent surgery. ${ }^{1,110-112}$

\section{New and future therapies for UC Vedolizumab}

Vedolizumab is a humanized monoclonal antibody targeting $\alpha 4 \beta 7$ integrin, a surface glycoprotein variably expressed on circulating B- and T-lymphocytes that interacts with intestinal mucosal addressin cell adhesion molecule. ${ }^{113}$ The drug selectively inhibits lymphocyte trafficking in the gut. Natalizumab, a monoclonal antibody with similar mechanism of action used to treat $C D$ and multiple sclerosis, blocks both $\alpha 4 \beta 7$ and $\alpha 4 \beta 1$ integrins; it works upon multiple organs including the brain and gut and carries the potential rare but serious and irreversible side effect of progressive multifocal leukoencephalopathy. ${ }^{114}$ Vedolizumab does not cross the blood-brain barrier and has not been shown to interfere with trafficking in the central nervous system. Two integrated randomized, double-blind, placebo-controlled trials demonstrated vedolizumab as a new and effective treatment for induction and maintenance of remission in active UC compared to placebo with 6 -week response rates of $47.1 \%$ in the vedolizumab group and $25.5 \%$ in the placebo group (95\% CI 11.6-31.7; $P<0.001) .{ }^{115}$ Induction dosing is $300 \mathrm{mg}$ IV at 0,2 , and 6 weeks. No significant difference in safety profiles was detected between the two groups particularly regarding serious infections. No cases of progressive multifocal leukoencephalopathy were reported. Few clinically important infusion reactions were seen, and there were no documented cases of anaphylaxis or serum sickness. Infection rates did not appear significantly different between 
groups. Further studies are needed to obtain long-term efficacy and safety data.

\section{Tofacitinib}

Tofacitinib is an oral agent that selectively inhibits the family of Janus tyrosine kinases 1-3. Janus tyrosine kinases are expressed in lymphoid cells and mediate signal transduction of multiple cytokines (such as interleukins 2, 4, 7, 9, 15, and 21) important for T-cell function such as lymphocyte activation and proliferation; blockade of the common signaling molecule shared by numerous cytokines should suppress B- and T-cells while maintaining regulatory T-cell function and leading to a state of immunosuppression or immunomodulation. ${ }^{115} \mathrm{~A}$ double-blind, placebo-controlled Phase II study evaluated the efficacy of tofacitinib in patients with moderate-tosevere UC. Clinical response as the primary outcome was achieved at 8 weeks and occurred with the highest rate in patients taking tofacitinib $15 \mathrm{mg}$ orally twice daily versus placebo (78\% versus 42\%; 95\% CI 66-89; $P<0.001$ ). Clinical remission rates were highest in the groups receiving tofacitinib $10 \mathrm{mg}$ twice daily (95\% CI 31-66; $P<0.001)$ and $15 \mathrm{mg}$ twice daily $(95 \% \mathrm{CI} 27-55 ; P<0.001)$ versus placebo. The most common side effects related to infection included nasopharyngitis and influenza. There was a dose-dependent increase in low-density and high-density lipoprotein cholesterol levels that reversed after drug cessation. Absolute neutrophil count was $<1,500$ cells/ $\mathrm{mm}^{3}$ in three patients while taking the therapy, but overall levels did not drop $<1,000$ cells $/ \mathrm{mm}^{3}$. ${ }^{116}$ The efficacy of tofacitinib as a maintenance therapy is unknown at this time, and further studies are needed to obtain long-term safety data.

\section{Patient-centered issues: QOL and medication compliance}

As a chronic disease, UC can significantly impact a patient's functional status and QOL. A significant reduction in both general life satisfaction and in health-related QOL scores have been documented in IBD particularly for patients exhibiting active disease compared to the general population and to IBD patients with quiescent disease. ${ }^{117}$ A large sample of 945 randomly selected IBD patients from the Crohn's and Colitis Foundation of America Partners Internet cohort completed a validated Patient Assessment of Chronic Illness Care (PACIC) instrument in order to assess perceptions of various facets of care within the Chronic Care Model. Scoring ranged from one to five, with five being the highest perception of care. The average PACIC score overall was 2.4 (standard deviation 0.93). Recent gastroenterology appointment, hospitalization, surgery, and current pouch/ostomy were associated with higher PACIC scores $(P<0.05)$. Positive correlation was seen with PACIC and QOL but not with adherence to medication or disease activity. Additionally, patients who had more subspecialist interactions had elevated perceptions of care, underscoring the importance of direct patient-physician connections. ${ }^{118}$

Medication compliance and patient education are important issues in IBD-related care. Nonadherence can be substantial, impacting disease activity and associated complications and contributing to both inpatient and outpatient health care costs. ${ }^{119,120}$ Factors associated with nonadherence or intermittent medication compliance include younger age, female sex, multiple daily pill burden, involvement of noncommercial payer or preferred provider organization insurance, nonuse of biologics/immunosuppressants/rectal 5-ASAs, and absence of specialist care. ${ }^{121}$

Nonadherence to 5-ASAs is particularly high with reported index medication compliance of $57.2 \%$ in one study. ${ }^{119}$ Greater risk of disease relapse has been seen in both nonadherent patients and in adherent patients whose mesalazine formulations were switched. ${ }^{122}$ Patients on single daily dose MMX mesalamine appear to have the lowest discontinuation risk and highest adherence rate. ${ }^{121}$ Over $75 \%$ of IBD patients are compliant with anti-TNF therapy. Factors predicting nonadherence for IFX or ADA include female sex, smoking, anxiety, moodiness, and treatment-related constraints. ${ }^{123}$ It has been reported that IBD patients may misperceive the risks and benefits of anti-TNF therapy, namely IFX; as per questionnaire results, a majority of IBD patients would be unwilling to accept treatment-related side effects if the remission rate at 1 year was $<75 \%$. Such perceptions appear to be independent of age and duration of disease. ${ }^{124}$ In one study, highest adherence rates were seen with immunomodulators. ${ }^{125}$

Medication compliance should be assessed during each patient evaluation, especially in the setting of quiescent disease. Stress and mental health-related disorders like anxiety and depression reflect health-related QOL in IBD patients and may affect compliance so should be addressed in conjunction with medical and/or surgical therapy. ${ }^{126}$ Development of a patient-administered tool to assist practitioners in evaluating noncompliance would be beneficial in the clinical setting. ${ }^{125}$ Web-based health care designs may improve patients' knowledge and QOL in the setting of chronic disease and may encourage adherence through a model of continued care. ${ }^{127}$

Practitioners should have a heightened sensitivity for the aforementioned patient-centered issues. Individualized 
therapeutic approaches are required and must take into account extent and severity of inflammatory disease with additional considerations for such factors as medication administration (route, pill burden), financial costs, age, lifestyle, occupation, and fertility/pregnancy status among others. Careful provision of informed consent relating to IBD therapy includes discussion of available therapeutic options, risks associated with lack of or suboptimal treatment and/or noncompliance, and pharmacotherapeutic alternatives such as colectomy. The ability of patients to comprehend and procure information about their chronic disease state is paramount. Shared decision making is an optimal model in such medical treatment encounters and should thus be advocated. ${ }^{128}$

\section{Conclusion}

$\mathrm{UC}$ is a chronic inflammatory colonic disease with a relapsing and remitting course. Once the diagnosis is established, new-onset cases should be stratified into mild, moderate, or severe disease categories to guide initial therapy. In general, mild-to-moderate UC can be managed in the outpatient setting with 5-ASAs, mesalamine, and topical corticosteroids (combined topical and rectal therapy ideal) and with oral corticosteroids in unresponsive cases. Oral or IV corticosteroids in the short-term are used for moderate UC with consideration of long-term options such as biologic agents or immunomodulators. Patients with moderate $\mathrm{UC}$, those recalcitrant to IV corticosteroids, or those with disease complications should be considered for a biologic agent or CsA; colectomy is also a rescue option for moderate-to-severe cases. Early surgical involvement is crucial, and colectomy may be life-saving in refractory or fulminant cases. Frequent assessments are needed to determine clinical response with intensification of therapy as needed to achieve disease control. Short-term treatment choices may be influenced by long-term management goals. Several new drugs have shown efficacy and safety for UC. Medication compliance and health-related QOL are important patient-centered issues. Future directions include continued drug development with longer-term safety and efficacy profiling and patient initiatives to encourage continued quality IBD care.

\section{Disclosure}

Dr Sunanda Kane is a consultant for AbbVie and Shire. Renée Marchioni Beery reports no conflicts of interest in this work.

\section{References}

1. Kornbluth A, Sachar DB. Ulcerative colitis practice guidelines in adults: American College of Gastroenterology, Practice Parameters Committee. Am J Gastroenterol. 2010;105(3):501-523.

2. Baumgart DC, Sandborn WJ. Inflammatory bowel disease: clinical aspects and established and evolving therapies. Lancet. 2007;369(9573): 1641-1657.
3. Molodecky NA, Soon IS, Rabi DM, et al. Increasing incidence and prevalence of the inflammatory bowel diseases with time, based on systematic review. Gastroenterology. 2012;142(1):46-54.

4. Kappelman MD, Moore KR, Allen JK, Cook SF. Recent trends in the prevalence of Crohn's disease and ulcerative colitis in a commercially insured US population. Dig Dis Sci. 2013;58(2):519-525.

5. Khalili H, Huang ES, Ananthakrishnan AN, et al. Geographical variation and incidence of inflammatory bowel disease among US women. Gut. 2012;61(12):1686-1692.

6. Munkholm P. Crohn's disease - occurrence, course and prognosis. An epidemiologic cohort-study. Dan Med Bull. 1997;44(3):287-302.

7. Samuel S, Ingle SB, Dhillon S, et al. Cumulative incidence and risk factors for hospitalization and surgery in a population-based cohort of ulcerative colitis. Inflamm Bowel Dis. 2013;19(9): 1858-1866.

8. Andres PG, Friedman LS. Epidemiology and the natural course of inflammatory bowel disease. Gastroenterol Clin North Am. 1999;28(2): 255-281,vii.

9. Bernstein CN, Ng SC, Lakatos PL, et al. A review of mortality and surgery in ulcerative colitis: milestones of the seriousness of the disease. Inflamm Bowel Dis. 2013;19(9):2001-2010.

10. Lutgens MW, van Oijen MG, van der Heijden GJ, Vleggaar FP, Siersema PD, Oldenburg B. Declining risk of colorectal cancer in inflammatory bowel disease: an updated meta-analysis of population-based cohort studies. Inflamm Bowel Dis. 2013;19(4): 789-799.

11. Cohen RD, Yu AP, Wu EQ, Xie J, Mulani PM, Chao J. Systematic review: the costs of ulcerative colitis in Western countries. Aliment Pharmacol Ther. 2010;31(7):693-707.

12. Kappelman MD, Rifas-Shiman SL, Porter CQ, et al. Direct health care costs of Crohn's disease and ulcerative colitis in US children and adults. Gastroenterology. 2008;135(6):1907-1913.

13. Nguyen GC, Tuskey A, Dassopoulos T, Harris ML, Brant SR. Rising hospitalization rates for inflammatory bowel disease in the United States between 1998 and 2004. Inflamm Bowel Dis. 2007;13(12): 1529-1535.

14. Pant C, Anderson MP, Deshpande A, et al. Trends in hospitalizations of children with inflammatory bowel disease within the United States from 2000 to 2009. J Investig Med. 2013;61(6):1036-1038.

15. Xavier RJ, Podolsky DK. Unraveling the pathogenesis of inflammatory bowel disease. Nature. 2007;448(7152):427-434.

16. International Inflammatory Bowel Disease Genetics Consortium (IIBDGC) [homepage on the Internet]. Hinxton: Wellcome Trust Sanger Institute; 2012 [updated October 25, 2013]. Available from: http://www. ibdgenetics.org. Accessed October 25, 2013.

17. Knights D, Lassen KG, Xavier RJ. Advances in inflammatory bowel disease pathogenesis: linking host genetics and the microbiome. Gut. 2013;62(10):1505-1510.

18. Farmer RG, Easley KA, Rankin GB. Clinical patterns, natural history, and progression of ulcerative colitis. A long-term follow-up of 1116 patients. Dig Dis Sci. 1993;38(6):1137-1146.

19. Ho GT, Mowat C, Goddard CJ, et al. Predicting the outcome of severe ulcerative colitis: development of a novel risk score to aid early selection of patients for second-line medical therapy or surgery. Aliment Pharmacol Ther. 2004;19(10):1079-1087.

20. Truelove SC, Witts LJ. Cortisone in ulcerative colitis; final report on a therapeutic trial. Br Med J. 1955;2(4947):1041-1048.

21. Schroeder KW, Tremaine WJ, Ilstrup DM. Coated oral 5-aminosalicylic acid therapy for mildly to moderately active ulcerative colitis. A randomized study. $N$ Engl J Med. 1987;317(26):1625-1629.

22. Rutgeerts P, Sandborn WJ, Feagan BG, et al. Infliximab for induction and maintenance therapy for ulcerative colitis. $N$ Eng J Med. 2005;353(23):2462-2476.

23. Nguyen GC, Kaplan GG, Harris ML, Brant SR. A national survey of the prevalence and impact of Clostridium difficile infection among hospitalized inflammatory bowel disease patients. Am J Gastroenterol. 2008;103(6):1443-1450. 
24. Murthy SK, Steinhart AH, Tinmouth J, Austin PC, Daneman N, Nguyen GC. Impact of Clostridium difficile colitis on 5-year health outcomes in patients with ulcerative colitis. Aliment Pharmacol Ther. 2012;36(11-12):1032-1039.

25. Kandiel A, Lashner B. Cytomegalovirus colitis complicating inflammatory bowel disease. Am J Gastroenterol. 2006;101(12): $2857-2865$

26. Carbonnel F, Lavergne A, Lemann M, et al. Colonoscopy of acute colitis. A safe and reliable tool for assessment of severity. Dig Dis Sci. 1994;39(7):1550-1557.

27. Terheggen G, Lanyi B, Schanz S, et al. Safety, feasibility, and tolerability of ileocolonoscopy in inflammatory bowel disease. Endoscopy. 2008;40(8):656-663.

28. Navaneethan U, Parasa S, Venkatesh PG, Trikudanathan G, Shen B. Prevalence and risk factors for colonic perforation during colonoscopy in hospitalized inflammatory bowel disease patients. J Crohns Colitis. 2011;5(3):189-195.

29. Navaneethan U, Kochhar G, Phull H, et al. Severe disease on endoscopy and steroid use increase the risk for bowel perforation during colonoscopy in inflammatory bowel disease patients. J Crohns Colitis. 2012;6(4):470-475.

30. Anupindi SA, Terreblanche O, Courtier J. Magnetic resonance enterography: inflammatory bowel disease and beyond. Magn Reson Imaging Clin NAm. 2013;21(4):731-750.

31. Ordas I, Rimola J, Garcia-Bosch O, et al. Diagnostic accuracy of magnetic resonance colonography for the evaluation of disease activity and severity in ulcerative colitis: a prospective study. Gut. 2013;62(11):1566-1572.

32. Pola S, Patel D, Ramamoorthy S, et al. Strategies for the care of adults hospitalized for active ulcerative colitis. Clin Gastroenterol Hepatol. 2012;10(12):1315-1325. e4.

33. Chang KH, Burke JP, Coffey JC. Infliximab versus cyclosporine as rescue therapy in acute severe steroid-refractory ulcerative colitis: a systematic review and meta-analysis. Int J Colorectal Dis. 2013;28(3): 287-293.

34. Herrinton LJ, Liu L, Fireman B, et al. Time trends in therapies and outcomes for adult inflammatory bowel disease, Northern California,1998-2005. Gastroenterology. 2009;137(2): 502-511.

35. Chande N. Prevention of venous thromboembolism in hospitalized patients with inflammatory bowel disease. Inflamm Bowl Dis. 2013;19(3):669-671.

36. Murthy SK, Nguyen GC. Venous thromboembolism in inflammatory bowel disease: an epidemiological review. Am J Gastroenterol. 2011;106(4):713-718

37. Fumery M, Xiaocang C, Dauchet L, Gower-Rousseau C, PeyrinBiroulet L, Colombel JF. Thromboembolic events and cardiovascular mortality in inflammatory bowel diseases: a meta-analysis of observational studies. J Crohns Colitis. Epub October 29, 2013.

38. Grainge MJ, West J, Card TR. Venous thromboembolism during active disease and remission in inflammatory bowel disease: a cohort study. Lancet. 2010;375(9715):657-663.

39. Nguyen GC, Sam J. Rising prevalence of venous thromboembolism and its impact on mortality among hospitalized inflammatory bowel disease patients. Am J Gastroenterol. 2008;103(9):2272-2280.

40. Wallaert JB, De Martino RR, Marsicovetere PS, et al. Venous thromboembolism after surgery for inflammatory bowel disease: are there modifiable risk factors? Data from ACS NSQIP. Dis Colon Rectum. 2012;55(11):1138-1144.

41. Ra G, Thanabalan R, Ratneswaran S, Nguyen GC. Predictors and safety of venous thromboembolism prophylaxis among hospitalized inflammatory bowel disease patients. J Crohns Colitis. 2013;7(10): e479-e485.

42. Marshall JK, Thabane M, Steinhart AH, Newman JR, Anand A, Irvine EJ. Rectal 5-aminosalicylic acid for induction of remission in ulcerative colitis [review]. Cochrane Database Syst Rev. 2010; 1:CD004115.
43. Gionchetti P, Rizzello F, Venturi A, et al. Comparison of oral with rectal mesalazine in the treatment of ulcerative proctitis. Dis Colon Rectum. 1998;41(1):93-97.

44. Marshall JK, Irvine EJ. Rectal corticosteroids versus alternative treatments in ulcerative colitis: a meta-analysis. Gut. 1997;40(6):775-781.

45. Cohen RD, Woseth DM, Thisted RA, Hanauer SB. A meta-analysis and overview of the literature on treatment options for left-sided ulcerative colitis and ulcerative proctitis. Am J Gastroenterol. 2000;95(5): 1263-1276.

46. Regueiro M, Loftus EV Jr, Steinhart AH, Cohen RD. Clinical guidelines for the management of left-sided ulcerative colitis and ulcerative proctitis: summary statement. Inflamm Bowel Dis. 2006;12(10):972-978.

47. Sandborn WJ, Hanauer SB. Systematic review: the pharmacokinetic profiles of oral mesalazine formulations and mesalazine pro-drugs used in the management of ulcerative colitis. Aliment Pharmacol Ther. 2003;17(1):29-42.

48. Kamm MA, Lichtenstein GR, Sandborn WJ, et al. Randomised trial of once- or twice-daily MMX mesalazine for maintenance of remission in ulcerative colitis. Gut. 2008;57(7):893-902.

49. Kamm MA, Lichtenstein GR, Sandborn WJ, et al. Effect of extended MMX mesalamine therapy for acute, mild-to-moderate ulcerative colitis. Inflamm Bowel Dis. 2009;15(1):1-8.

50. Flourie B, Hagege H, Tucat G, et al. Randomised clinical trial: once- vs twice-daily prolonged-release mesalazine for active ulcerative colitis. Aliment Pharmacol Ther. 2013;37(8):767-775.

51. Sandborn WJ, Regula J, Feagan BG, et al. Delayed-release oral mesalamine $4.8 \mathrm{~g} /$ day ( $800-\mathrm{mg}$ tablet) is effective for patients with moderately active ulcerative colitis. Gastroenterology. 2009;137(6):1934-1943.

52. Kane S, Huo D, Magnanti K. A pilot feasibility study of once daily versus conventional dosing mesalamine for maintenance of ulcerative colitis. Clin Gastroenterol Hepatol. 2003;1(3):170-173.

53. Raedler A, Behrens C, Bias P. Mesalazine (5-aminosalicylic acid) micropellets show similar efficacy and tolerability to mesalazine tablets in patients with ulcerative colitis - results from a randomized-controlled trial. Aliment Pharmacol Ther. 2004;20(11-12):1353-1363.

54. Dignass AU, Bokemeyer B, Adamek H, et al. Mesalamine once daily is more effective than twice daily in patients with quiescent ulcerative colitis. Clin Gastroenterol Hepatol. 2009;7(7):762-769.

55. Sandborn WJ, Korzenik J, Lashner B, et al. Once-daily dosing of delayed-release oral mesalamine (400-mg tablet) is as effective as twice-daily dosing for maintenance of remission of ulcerative colitis. Gastroenterology. 2010;138(4):1286-1296.

56. Sandborn WJ, Kamm MA, Lichtenstein GR, Lyne A, Butler T, Joseph RE. MMX Multi Matrix System mesalazine for the induction of remission in patients with mild-to-moderate ulcerative colitis: a combined analysis of two randomized, double-blind, placebocontrolled trials. Aliment Pharmacol Ther. 2007;26(2):205-215.

57. Safdi M, DeMicco M, Sninsky C, et al. A double-blind comparison of oral versus rectal mesalamine versus combination therapy in the treatment of distal ulcerative colitis. Am J Gastroenterol. 1997;92(10): 1867-1871.

58. Marteau P, Probert CS, Lindgren S, et al. Combined oral and enema treatment with Pentasa (mesalazine) is superior to oral therapy alone in patients with extensive mild/moderate active ulcerative colitis: a randomised, double blind, placebo controlled study. Gut. 2005;54(7): 960-965.

59. Campieri M, De Franchis R, Bianchi Porro G, Ranzi T, Brunetti G, Barbara L. Mesalazine (5-aminosalicylic acid) suppositories in the treatment of ulcerative proctitis or distal proctosigmoiditis. A randomized controlled trial. Scand J Gastroenterol. 1990;25(7):663-668.

60. Lamet M, Ptak T, Dallaire C, et al. Efficacy and safety of mesalamine $1 \mathrm{~g}$ HS versus $500 \mathrm{mg}$ BID suppositories in mild to moderate ulcerative proctitis: a multicenter randomized study. Inflamm Bowel Dis. 2005;11(7):625-630.

61. Sutherland LR, Martin F, Greer S, et al. 5-Aminosalicylic acid enema in the treatment of distal ulcerative colitis, proctosigmoiditis, and proctitis. Gastroenterology. 1987;92(6):1894-1898. 
62. Hanauer SB; US PENTASA Study Group. Dose-ranging study of mesalamine (PENTASA) enemas in the treatment of acute ulcerative proctosigmoiditis: results of a multicentered placebo-controlled trial. Inflamm Bowel Dis. 1998;4(2):79-83.

63. Ardizzone S, Doldo P, Ranzi T, et al; SAF-3 Study Group. Mesalazine foam (Salofalk foam) in the treatment of active distal ulcerative colitis. A comparative trial vs Salofalk enema. Ital J Gastroenterol Hepatol. 1999;31(8):677-684

64. Biancone L, Gionchetti P, Del Vecchio Blanco G, et al. Beclomethasone dipropionate versus mesalazine in distal ulcerative colitis: a multicenter, randomized, double-blind study. Dig Liver Dis. 2007;39(4):329-337.

65. Hanauer SB, Robinson M, Pruitt R, et al; US Budesonide Enema Study Group. Budesonide enema for the treatment of active, distal ulcerative colitis and proctitis: a dose-ranging study. Gastroenterology. 1998;115(3):525-532.

66. Sandborn WJ, Travis S, Moro L, et al. Once-daily budesonide $\mathrm{MMX}^{\circledR}$ extended-release tablets induce remission in patients with mild to moderate ulcerative colitis: results from the CORE I study. Gastroenterology. 2012;143(5):1218-1226.

67. Travis SP, Danese S, Kupcinskas L, et al. Once-daily budesonide MMX in active, mild-to-moderate ulcerative colitis: results from the randomised CORE II study. Gut. 2014;63(3):433-441.

68. Feagan BG, Macdonald JK. Oral 5-aminosalicylic acid for induction of remission in ulcerative colitis [review]. Cochrane Database Syst Rev. 2012;10:CD000543.

69. Sutherland L, Macdonald JK. Oral 5-aminosalicylic acid for induction of remission in ulcerative colitis [review]. Cochrane Database Syst Rev. 2006;2:CD000543.

70. Baron JH, Connell AM, Kanaghinis TG, Lennard-Jones JE, Jones AF. Out-patient treatment of ulcerative colitis. Comparison between three doses of oral prednisone. Br Med J. 1962;2(5302):441-443.

71. Turner D, Walsh CM, Steinhart AH, Griffiths AM. Response to corticosteroids in severe ulcerative colitis: a systematic review of the literature and a meta-regression. Clin Gastroenterol Hepatol. 2007;5(1):103-110.

72. Tourner M, Loftus EV Jr, Harmsen WS, et al. Risk factors for opportunistic infections in patients with inflammatory bowel disease. Gastroenterology. 2008;134(4):929-936.

73. Gisbert JP, Linares PM, McNicholl AG, Mate J, Gomollon F. Metaanalysis: the efficacy of azathioprine and mercaptopurine in ulcerative colitis. Aliment Pharmacol Ther. 2009;30(2):126-137.

74. Ardizzone S, Maconi G, Russo A, Imbesi V, Colombo E, Bianchi Porro G. Randomised controlled trial of azathioprine and 5-aminosalicylic acid for treatment of steroid dependent ulcerative colitis. Gut. 2006;55(1): 47-53.

75. Relling MV, Gardner EE, Sandborn WJ, et al. Clinical Pharmacogenetics Implementation Consortium guidelines for thiopurine methyltransferase genotype and thiopurine dosing. Clin Pharmacol Ther. 2011;89(3):387-391

76. Sands BE, Tremaine WJ, Sandborn WJ, et al. Infliximab in the treatment of severe, steroid-refractory ulcerative colitis: a pilot study. Inflamm Bowel Dis. 2001;7(2):83-88.

77. Jarnerot G, Hertervig E, Friis-Liby I, et al. Infliximab as a rescue therapy in severe to moderately severe ulcerative colitis: a randomized placebocontrolled study. Gastroenterology. 2005;128(7):1805-1811.

78. Reinisch W, Sandborn WJ, Hommes DW, et al. Adalimumab for induction of clinical remission in moderately to severely active ulcerative colitis: results of a randomised controlled trial. Gut. 2011;60(6): 780-787.

79. Sandborn WJ, van Assche G, Reinisch W, et al. Adalimumab induces and maintains clinical remission in patients with moderate-to-severe ulcerative colitis. Gastroenterology. 2012;142(2):257-265.

80. Feagan BG, Sandborn WJ, Lazar A, et al. Adalimumab therapy is associated with reduced risk of hospitalization in patients with ulcerative colitis. Gastroenterology. 2014;146(1):110-118

81. Sandborn WJ, Feagan BG, Marano C, et al. Subcutaneous golimumab induces clinical response and remission in patients with moderate-tosevere ulcerative colitis. Gastroenterology. 2014;146(1):85-95.
82. Sandborn WJ, Feagan BG, Marano C, et al. Subcutaneous golimumab maintains clinical response in patients with moderate-to-severe ulcerative colitis. Gastroenterology. 2014;146(1):96-109.

83. Dinesen LC, Walsh AJ, Protic MN, et al. The pattern and outcome of acute severe colitis. J Crohns Colitis. 2010;4(4):431-437.

84. Travis SP, Farrant JM, Ricketts C, et al. Predicting outcome in severe ulcerative colitis. Gut. 1996;38(6):905-910.

85. Aceituno M, García-Planella E, Heredia C, et al. Steroid-refractory ulcerative colitis: predictive factors of response to cyclosporine and validation in an independent cohort. Inflamm Bowel Dis. 2008;14(3): 347-352.

86. Message L, Bourreille A, Laharie D, et al. Efficacy of intravenous cyclosporin in moderately severe ulcerative colitis refractory to steroids. Gastroenterol Clin Biol. 2005;29(3):231-235.

87. Sood A, Midha V, Sood N, et al. Cyclosporine in the treatment of severe steroid refractory ulcerative colitis: a retrospective analysis of 24 cases. Indian J Gastroenterol. 2008;27(6):232-235.

88. Actis GC, Volpes R, Rizzetto M. Oral microemulsion cyclosporin to reduce steroids rapidly in chronic active ulcerative colitis. Eur $J$ Gastrenterol Hepatol. 1999;11(8):905-908.

89. Lichtiger S, Present DH, Kornbluth A, et al. Cyclosporine in severe ulcerative colitis refractory to steroid therapy. $N$ Engl J Med. 1994;330(26):1841-1845.

90. Ogata $\mathrm{H}$, Matsui T, Nakamura $\mathrm{M}$, et al. A randomized dose finding study of oral tacrolimus (FK506) therapy in refractory ulcerative colitis. Gut. 2006;55(9):1255-1262.

91. Benson A, Barrett T, Sparberg M, Buchman AL. Efficacy and safety of tacrolimus in refractory ulcerative colitis and Crohn's disease: a single-center experience. Inflamm Bowel Dis. 2008;14(1):7-12.

92. Baumgart DC, Pintoffl JP, Sturm A, Wiedenmann B, Dignass AU. Tacrolimus is safe and effective in patients with severe steroidrefractory or steroid-dependent inflammatory bowel disease - a long-term follow-up. Am J Gastroenterol. 2006;101(5):1048-1056.

93. Fellermann K, Ludwig D, Stahl M, David-Walek T, Stange EF. Steroid-unresponsive acute attacks of inflammatory bowel disease: immunomodulation by tacrolimus (FK506). Am J Gastroenterol. 1998;93(10):1860-1866

94. Schmidt KJ, Herrlinger KR, Emmrich J, et al. Short-term efficacy of tacrolimus in steroid-refractory ulcerative colitis - experience in 130 patients. Aliment Pharmacol Ther. 2013;37(1):129-136.

95. Leblanc S, Allez M, Seksik P, et al. Successive treatment with cyclosporine and infliximab in steroid-refractory ulcerative colitis. Am J Gastroenterol. 2011;106(4):771-777.

96. Ananthakrishnan AN, Issa M, Beaulieu DB, et al. History of medical hospitalization predicts future need for colectomy in patients with ulcerative colitis. Inflamm Bowel Dis. 2009;15(2):176-181.

97. Tøttrup A, Erichsen R, Svaerke C, Laurberg S, Srensen HT. Thirty-day mortality after elective and emergency total colectomy in Danish patients with inflammatory bowel disease: a population-based nationwide cohort study. BMJ Open. 2012;2(2):e000823.

98. Coakley BA, Telem D, Nguyen S, Dallas K, Divino CM. Prolonged preoperative hospitalization correlates with worse outcomes after colectomy for acute fulminant ulcerative colitis. Surgery. 2013;153(2):242-248.

99. Grucela A, Steinhagen RM. Current surgical management of ulcerative colitis. Mt Sinai J Med. 2009;76(6):606-612.

100. Cohen JL, Strong SA, Hyman NH, et al. Practice parameters for the surgical treatment of ulcerative colitis. Dis Colon Rectum. 2005;48(11): 1997-2009.

101. Hicks CW, Hodin RA, Bordeianou L. Possible overuse of 3-stage procedures for active ulcerative colitis. JAMA Surg. 2013;148(7): 658-664.

102. Burke DA, Axon AT, Clayden SA, Dixon MF, Johnston D, Lacey RW. The efficacy of tobramycin in the treatment of ulcerative colitis. Aliment Pharmacol Ther. 1990;4(2):123-129.

103. Lobo AJ, Burke DA, Sobala GM, Axon AT. Oral tobramycin in ulcerative colitis: effect on maintenance of remission. Aliment Pharmacol Ther. 1993;7(2):155-158. 
104. Turunen UM, Farkkila MA, Hakala K, et al. Long-term treatment of ulcerative colitis with ciprofloxacin: a prospective, doubleblind, placebo-controlled study. Gastroenterology. 1998;115(5): 1072-1078.

105. Mantzaris GJ, Archavlis E, Christoforidis P, et al. A prospective randomized controlled trial of oral ciprofloxacin in acute ulcerative colitis. Am J Gastroenterol. 1997;92(3):454-456.

106. Mantzaris GJ, Petraki K, Archavlis E, et al. A prospective randomized controlled trial of intravenous ciprofloxacin as an adjunct to corticosteroids in acute, severe ulcerative colitis. Scand $J$ Gastroenterol. 2001;36(9):971-974.

107. Dickinson RJ, O’Connor HJ, Pinder I, Hamilton I, Johnston D, Axon AT. Double blind controlled trial of oral vancomycin as adjunctive treatment in acute exacerbations of idiopathic colitis. Gut. 1985;26(12): $1380-1384$.

108. Mantzaris GJ, Hatzis A, Kontogiannis P, Triadaphyllou G. Intravenous tobramycin and metronidazole as an adjunct to corticosteroids in acute, severe ulcerative colitis. Am J Gastroenterol. 1994;89(1):43-46.

109. Chapman RW, Selby WS, Jewell DP. Controlled trial of intravenous metronidazole as an adjunct to corticosteroids in severe ulcerative colitis. Gut. 1986;27(10):1210-1212.

110. Truelove SC, Jewell DP. Intensive intravenous regimen for severe attacks of ulcerative colitis. Lancet. 1974;1(7866):1067-1070.

111. Truelove SC, Willoughby CP, Lee EG, Kettlewell MG. Further experience in the treatment of severe attacks of ulcerative colitis. Lancet. 1978;2(8099):1086-1088.

112. Jarnerot G, Rolny P, Sandberg-Gertzen H. Intensive intravenous treatment of ulcerative colitis. Gastroenterology. 1985;89(5): 1005-1013.

113. Feagan BG, Rutgeerts $\mathrm{P}$, Sands BE, et al. Vedolizumab as induction and maintenance therapy for ulcerative colitis. $N \mathrm{Engl} \mathrm{J} \mathrm{Med.}$ 2013;369(8):699-710.

114. Schwab N, Hohn KG, Schneider-Hohendorf T, et al. Immunological and clinical consequences of treating a patient with natalizumab. Mult Scler. 2012;18(3):335-344.

115. Flanagan ME, Blumenkopf TA, Brissette WH, et al. Discovery of CP-690,550: a potent and selective Janus kinase (JAK) inhibitor for the treatment of autoimmune diseases and organ transplant rejection. J Med Chem. 2010;53(24):8468-8484.

116. Sandborn WJ, Ghosh S, Panes J, et al. Tofacitinib, an oral Janus kinase inhibitor, in active ulcerative colitis. $N$ Engl J Med. 2012;367(7): 616-624.

117. Janke KH, Raible A, Bauer M, et al. Questions on life satisfaction (FLZM) in inflammatory bowel disease. Int $J$ Colorectal Dis. 2004;19(4):343-353.
118. Randell RL, Long MD, Martin CF, et al. Patient perception of chronic illness care in a large inflammatory bowel disease cohort. Inflamm Bowel Dis. 2013;19(7):1428-1433.

119. Kane S, Shaya F. Medication non-adherence is associated with increased medical health care costs. Dig Dis Sci. 2008;53(4):1020-1024.

120. Mitra D, Hodgkins P, Yen L, Davis KL, Cohen RD. Association between oral 5-ASA adherence and health care utilization and costs among patients with active ulcerative colitis. BMC Gastroenterol. 2012;12:132.

121. Yen L, Wu J, Hodgkins PL, Cohen RD, Nichol MB. Medication use patterns and predictors of nonpersistence and nonadherence with oral 5-aminosalicylic acid therapy in patients with ulcerative colitis. J Manag Care Pharm. 2012;18(9):701-712.

122. Robinson A, Hankins M, Wiseman G, Jones M. Maintaining stable symptom control in inflammatory bowel disease: a retrospective analysis of adherence, medication switches and the risk of relapse. Aliment Pharmacol Ther. 2013;38(5):531-538.

123. Lopez A, Billioud V, Peyrin-Biroulet C, Peyrin-Biroulet L. Adherence to anti-TNF therapy in inflammatory bowel diseases: a systematic review. Inflamm Bowel Dis. 2013;19(7):1528-1533.

124. Baars JE, Siegel CA, Kuipers EJ, van der Woude CJ. Patient's perspectives important for early anti-tumor necrosis factor treatment in inflammatory bowel disease. Digestion. 2009;79(1):30-35.

125. Kane S, Becker B, Harmsen WS, Kurian A, Morisky DE, Zinsmeister AR. Use of a screening tool to determine nonadherent behavior in inflammatory bowel disease. Am J Gastroenterol. 2012; 107(2):154-160.

126. Iglesias-Rey M, Barreiro-de Acosta M, Caamano-Isorna F, et al. Psychological factors are associated with changes in the health-related quality of life in inflammatory bowel disease. Inflamm Bowel Dis. 2014;20(1):92-102.

127. Elkjaer M, Shuhaibar M, Burisch J, et al. E-health empowers patients with ulcerative colitis: a randomised controlled trial of the web-guided "Constant-care" approach. Gut. 2010;59(12):1652-1661.

128. Charles C, Gafni A, Whelan T. Shared decision-making in the medical encounter: what does it mean? (or it takes at least two to tango). Soc Sci Med. 1997;44(5):681-692.

129. Lichtenstein GR, Abreu MT, Cohen R, Tremaine W. American Gastroenterological Association Institute technical review on corticosteroids, immunomodulators, and infliximab in inflammatory bowel disease. Gastroenterology. 2006;130(3):940-987.
Clinical and Experimental Gastroenterology

\section{Publish your work in this journal}

Clinical and Experimental Gastroenterology is an international, peerreviewed, open access journal, publishing all aspects of gastroenterology in the clinic and laboratory, including: Pathology, pathophysiology of gastrointestinal disease; Investigation and treatment of gastointestinal disease; Pharmacology of drugs used in the alimentary tract;

\section{Dovepress}

Immunology/genetics/genomics related to gastrointestinal disease. This journal is indexed on CAS. The manuscript management system is completely online and includes a very quick and fair peer-review system. Visit http://www.dovepress.com/testimonials.php to read real quotes from published authors. 